\title{
BLACK HOLE-NEUTRON STAR MERGERS WITH A HOT NUCLEAR EQUATION OF STATE: OUTFLOW AND NEUTRINO-COOLED DISK FOR A LOW-MASS, HIGH-SPIN CASE
}

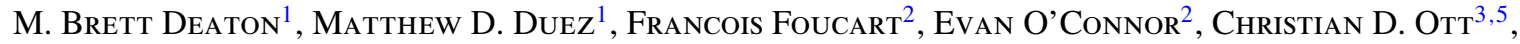 \\ Lawrence E. Kidder ${ }^{4}$, Curran D. Muhlberger ${ }^{4}$, Mark A. Scheel ${ }^{3}$, and Bela Szilagyi ${ }^{3}$ \\ ${ }^{1}$ Department of Physics and Astronomy, Washington State University, Pullman, WA 99164, USA; mbdeaton@wsu.edu, m.duez@wsu.edu \\ ${ }^{2}$ Canadian Institute for Theoretical Astrophysics, University of Toronto, Toronto, Ontario M5S 3H8, Canada \\ ${ }^{3}$ TAPIR, MC 350-17, California Institute of Technology, Pasadena, CA 91125, USA \\ ${ }^{4}$ Center for Radiophysics and Space Research, Cornell University, Ithaca, NY 14853, USA \\ Received 2013 April 25; accepted 2013 September 4; published 2013 September 24
}

\begin{abstract}
Neutrino emission significantly affects the evolution of the accretion tori formed in black hole-neutron star mergers. It removes energy from the disk, alters its composition, and provides a potential power source for a gamma-ray burst. To study these effects, simulations in general relativity with a hot microphysical equation of state (EOS) and neutrino feedback are needed. We present the first such simulation, using a neutrino leakage scheme for cooling to capture the most essential effects and considering a moderate mass $\left(1.4 M_{\odot}\right.$ neutron star, $5.6 M_{\odot}$ black hole), high-spin (black hole $J / M^{2}=0.9$ ) system with the $K_{0}=220 \mathrm{MeV}$ Lattimer-Swesty EOS. We find that about $0.08 M_{\odot}$ of nuclear matter is ejected from the system, while another $0.3 M_{\odot}$ forms a hot, compact accretion disk. The primary effects of the escaping neutrinos are (1) to make the disk much denser and more compact, (2) to cause the average electron fraction $Y_{e}$ of the disk to rise to about 0.2 and then gradually decrease again, and (3) to gradually cool the disk. The disk is initially hot $(T \sim 6 \mathrm{MeV})$ and luminous in neutrinos $\left(L_{v} \sim 10^{54} \mathrm{erg} \mathrm{s}^{-1}\right)$, but the neutrino luminosity decreases by an order of magnitude over $50 \mathrm{~ms}$ of post-merger evolution.
\end{abstract}

Key words: black hole physics - gamma-ray burst: general - neutrinos - stars: neutron

Online-only material: color figures

\section{INTRODUCTION}

Much of the interest in black hole-neutron star (BHNS) mergers arises from their potential to solve two important problems in contemporary astrophysics. First, it is possible that such events can produce short hard gamma-ray bursts (SGRBs). Second, they could significantly contribute to the abundances of $r$-process nuclei observed in the solar system.

SGRBs emit characteristic luminosities of $L \sim 10^{50-52} /$ $(4 \pi) \mathrm{erg} \mathrm{s}^{-1}$ steradian $^{-1}$, with typical durations of $\sim 1 \mathrm{~s}$, and peak photon energies of $h v \sim 1 \mathrm{MeV}$. Their spectra are nonthermal, and vary in brightness on a timescale of $\Delta t \lesssim 10 \mathrm{~ms}$ (Nakar 2007). Combined observations of a nonthermal spectrum and a short time variability can be explained by an ultrarelativistic jet $(\Gamma \gtrsim 30)$ launched from a disk experiencing rapid accretion onto a black hole (Narayan et al. 1992; Nakar 2007). BHNS mergers are plausible progenitors for such a system.

BHNS mergers may also produce unbound neutron-rich ejecta, which would provide an $r$-process nucleosynthesis site needed to explain the observed heavy element abundances (Lattimer \& Schramm 1974; Freiburghaus et al. 1999; Arnould et al. 2007; Korobkin et al. 2012). Additionally such outflows may be observable from the radioactive decay that results after unstable heavy isotopes are formed (a "kilonova") or from the shock that would form when the outflow hits a sufficiently dense interstellar medium (Metzger \& Berger 2012).

BHNS mergers and their aftermath involve relativistic gravity, magnetohydrodynamics, nuclear physics, and neutrino radiation. In addition, a large space of binary parameters must be explored, since a wide range of premerger black hole mass and spin values are plausible (Belczynski et al. 2008; Özel et al. 2010). Early attempts to explore this parameter space in general

\footnotetext{
5 Alfred P. Sloan Research Fellow.
}

relativity have used polytropic (Taniguchi et al. 2005; Shibata \& Uryu 2006; Etienne et al. 2008, 2009; Duez et al. 2008, 2010; Foucart et al. 2011, 2012; Etienne et al. 2012; Foucart et al. 2013) or piecewise-polytropic (Kyutoku et al. 2010, 2011; Lackey et al. 2013) EOSs to describe the neutron star matter, with the thermal effects being modeled by a simple $\Gamma$-law. Because the matter does not heat until after tidal disruption, use of these $\Gamma$-law EOSs may be adequate for the inspiral and very early tidal disruption phases, which produce nearly all of the gravitational wave signal. From these parameter space studies, a general picture has begun to emerge (Faber 2009; Duez 2010; Pannarale et al. 2011; Shibata \& Taniguchi 2011; Foucart 2012). In the best-understood binary parameter space of spin $\left(a^{*} \equiv\left(J_{\mathrm{BH}} / M_{\mathrm{BH}}^{2}\right)(c / G)=[-0.5,0.9]\right)$, neutron star compactness $\left(\mathcal{C} \equiv\left(M_{\mathrm{NS}} / R_{\mathrm{NS}}\right)\left(G / c^{2}\right) \sim[0.13,0.20]\right)$, and mass ratio $\left(q \equiv M_{\mathrm{BH}} / M_{\mathrm{NS}} \sim[1,7]\right)$-where $\mathrm{G}$ is Newton's gravitational constant and $\mathrm{c}$ is the speed of light—disks of significant masses appear to be formed in binaries with low mass ratios, low neutron star compactness, or high prograde black hole spins.

After the merger, neutrinos will begin to play an important role in the evolution. Following disruption, the fluid is shocked to temperatures of $\sim 10 \mathrm{MeV}$ and begins to radiate copiously. In simulations of accretion disk-black hole systems (Shibata \& Sekiguchi 2012), and binary neutron star mergers (Ruffert et al. 1997; Rosswog \& Liebendörfer 2003; Sekiguchi et al. 2011), which produce disks similar to those of BHNS mergers, total neutrino luminosities are of order $10^{51-53} \mathrm{erg} \mathrm{s}^{-1}$. BHNS simulations using $\Gamma$-law EOSs indicate that disks last at most several hundred milliseconds (Etienne et al. 2009; Duez et al. 2010; Kyutoku et al. 2010; Foucart et al. 2011). During this time, neutrinos may carry away a significant amount of energy from the accretion disk, causing it to cool and contract. Additionally, neutrinos, unlike photons, change the composition of their source. In the present case, initially neutron-rich neutron star 
material releptonizes as it expands into a disk through an imbalance of electron- and positron-capture reactions. These composition changes can play a significant role in BHNS postmerger dynamics in a number of ways. Changes to the electron fraction alter the luminosity of each neutrino species. Lepton number gradients affect pressure forces and can drive convection (Lee et al. 2005). Phase transitions, e.g., recombination from pure nucleonic matter into alpha particles and heavy nuclei, can release or absorb energy as density and temperature change. A temperature- and composition-dependent EOS is needed to capture all of these effects.

Neutrino emission may also play a crucial role in powering the relativistic outflow needed for an SGRB. It could do this by depositing energy in the funnel region along the $\mathrm{BH}$ spin axis via neutrino pair annihilation $\left(v \bar{v} \rightarrow e^{-} e^{+}\right)$. Depending on the shape of the emitting region, its proximity to the black hole, and its emission spectrum, the annihilation process could proceed with an efficiency as high as $0.2 \%-0.5 \%$ (Ruffert et al. 1997; Birkl et al. 2007; Dessart et al. 2009; Harikae et al. 2010). This would indicate that neutrino pair annihilation may only be able to power low-energy SGRBs. However, accurate relativistic simulations of the fluid and neutrino fields are needed to make reliable statements about the efficiency of the neutrino pair annihilation process in BHNS mergers.

Because the disk spans optically thick and optically thin regimes, a full 6-dimensional evolution of the neutrino fields ( 1 energetic +3 spatial +2 angular dimensions) is needed to completely describe the coupling between radiation and matter. This is impossible with current resources. Fortunately, many of the essential features of the radiation can be captured using a simple leakage scheme. Rather than performing actual radiation transport, leakage schemes remove energy and alter lepton number at rates based on the local free-emission and diffusion rates. Leakage certainly neglects some arguably important effects (e.g., neutrino absorption-driven winds), but it captures the basic energetics and composition drift of the post-merger system. (See e.g., Ott et al. 2012 for an analysis of leakage in core-collapse supernovae simulations.)

In Newtonian gravity, microphysical EOSs and neutrino leakage schemes have been used in simulations of binary neutron star (Ruffert et al. 1996, 1997; Rosswog \& Davies 2002; Rosswog \& Liebendörfer 2003) and BHNS (Janka et al. 1999; Rosswog et al. 2004) mergers. Leakage schemes have recently been incorporated into three-dimensional general relativistic simulations of stellar core collapse (Sekiguchi \& Shibata 2011; Ott et al. 2012) and binary neutron star mergers (Sekiguchi et al. 2011; Kiuchi et al. 2012). Simulations of BHNS mergers with a microphysical EOS but no neutrino feedback were presented by Duez et al. (2010). The first relativistic BHNS simulations with both a microphysical EOS and neutrino feedback are presented here.

In this paper, we introduce a neutrino leakage scheme into the Spectral Einstein Code, ${ }^{6}$ evolving the coupled hydrodynamics-Einstein field equations with leakage, and apply it to one BHNS case, a moderately low-mass $\left(M_{\mathrm{NS}}=1.4 M_{\odot}\right.$, $\left.M_{\mathrm{BH}}=5.6 M_{\odot}\right)$, high black hole spin $\left(a^{*}=0.9\right)$ system. We follow six orbits of inspiral and evolve for $50 \mathrm{~ms}$ past merger. We employ the finite-temperature EOS of Lattimer \& Swesty (1991) using a compressibility parameter $K_{0}=220 \mathrm{MeV}$ that yields neutron stars consistent with existing observations (see e.g., Demorest et al. 2010 and Steiner et al. 2010 — though recent work by Guillot et al. 2013 predicts smaller neutron star radii).

\footnotetext{
6 http://www.black-holes.org/SpEC.html
}

We find an initially large post-merger disk of mass $M_{0} \sim$ $0.3 M_{\odot}$ which accretes rapidly for the first $30 \mathrm{~ms}$ and then settles to a slow mass depletion rate, from which we can estimate a disk lifetime of $T_{\text {dep }} \gtrsim 0.2 \mathrm{~s}$. The neutrino luminosity peaks $10 \mathrm{~ms}$ after merger at $\sim 10^{54} \mathrm{erg} \mathrm{s}^{-1}$ but drops to $\sim 2 \times 10^{53} \mathrm{erg} \mathrm{s}^{-1}$ by the end of the $50 \mathrm{~ms}$ post-merger evolution, at which time it continues to drop. Assuming a conservative efficiency of $0.2 \%$ (Ruffert et al. 1997), we estimate that the peak work done by neutrino pair annihilation could be as high as $Q_{\nu \bar{v}} \sim 10^{51} \mathrm{erg} \mathrm{s}^{-1}$. We also find a large unbound outflow from the system, of mass $M_{\mathrm{ej}} \sim 0.08 M_{\odot}$, with mildly relativistic velocities, leading to an available kinetic energy that could be as high as $E_{\text {ej }} \sim 10^{52} \mathrm{erg}$.

The disk itself displays an interesting evolution during this time. A very high energy region-both hot and having superKeplerian kinetic energies-develops in the innermost disk. This configuration is unstable, and nonaxisymmetric perturbations persist in the inner disk for many dynamical times. Away from the edges, the disk does roughly settle to stationary axisymmetry, and evolution is driven primarily by secular radiation and disk depletion effects. Neutrino cooling leads to a much higherdensity, and somewhat lower-entropy, disk structure than seen in a comparison run without cooling. The average temperature decreases in the disk from a maximum of $\sim 6 \mathrm{MeV}$ at $15 \mathrm{~ms}$ after merger to $\sim 4 \mathrm{MeV}$ at $50 \mathrm{~ms}$. The average specific entropy $\left(\sim 0.1 k_{\mathrm{B}}\right.$ baryon $^{-1}$ during inspiral) stays near $\sim 8 k_{\mathrm{B}}$ baryon $^{-1}$ from $5 \mathrm{~ms}$ to the end of the evolution. The disk is sufficiently hot and dense to remain opaque to neutrinos of all species. The average electron fraction rises from 0.07 in the initial neutron star to around 0.2 at $20 \mathrm{~ms}$ after merger. At this time, electron neutrino and antineutrino number emission roughly balance. Afterward, the electron fraction decreases gradually as the disk cools and the balance of $v_{e}$ and $\bar{v}_{e}$ emission adjusts. The disk continues to cool and flatten, so that by the end of our simulation its luminosity in neutrinos has fallen by an order of magnitude. Longer-lasting high-power energy release may come from physics not included in this simulation, particularly magnetic fields.

This paper is organized as follows. In Section 2, we discuss the numerical methods used to simulate the mergers; in Sections 2.1-2.3, we describe our treatment of the nuclear EOS, initial data, and neutrino leakage, respectively. In Section 3, we give a summary of the evolution, examining several measures of convergence. In Section 4, we analyze outflows. In Section 5, we review the relevant timescales for the accretion disk. In Sections 6 and 7, we analyze the accretion disk in the epochs of formation and neutrino-driven evolution, respectively. The importance of neutrino cooling effects in general is demonstrated in Section 8, in which we compare the results of simulations performed with and without neutrino leakage effects. We summarize our conclusions in Section 9.

Throughout the rest of this paper, unless otherwise noted, we adopt geometric units in which $G=c=1$, and we use Latin indices $(j, k)$ to represent the three spatial coordinates, and Greek indices $(\alpha, \beta)$ to represent the four spacetime coordinates. Unless a different average is specified, angle brackets around thermodynamic quantities indicate a density-weighted average (e.g., $\left.\left\langle Y_{e}\right\rangle \equiv \int Y_{e} \rho d^{3} x / \int \rho d^{3} x\right)$.

\section{METHODS}

We evolve the coupled general relativistic-hydrodyamics system using the Spectral Einstein Code (SpEC). We 
employ our standard two-grid pseudospectral/finite-difference approach, described in detail in earlier papers (Scheel et al. 2006; Duez et al. 2008; Hemberger et al. 2013; Foucart et al. 2013) and briefly summarized here. The spacetime is described by the 4-metric and its time derivative, which we evolve in the generalized harmonic formulation using a multidomain pseudospectral algorithm (Lindblom et al. 2006). The coordinates are evolved by enforcing the "frozen" gauge condition described in Appendix A.1 of Foucart et al. (2013). The fluid is described by the hydrodynamic fields, which we evolve in conservative form using shock-capturing finite-difference methods. The metric and fluid are evolved on separate computational domains; fluid source terms for the metric evolution and metric source terms for the fluid evolution are acquired by interpolation between domains. The fluid domain is a uniform Cartesian grid covering the non-vacuum upper hemisphere, with the lower hemisphere fluid quantities set by an assumed equatorial symmetry. The black hole singularity is handled by excising a region inside the apparent horizon, i.e., by not placing colocation points there. The fluid grid does have points inside the excised region, but the fields at these points are not evolved, and one-sided stencils are used to evolve points next to the excision boundary.

The fluid is described by the baryonic rest mass density $\rho$, temperature $T$, electron fraction (electrons per baryon) $Y_{e}$, and 3 -velocity $v_{i}$. The pressure $P$ and specific internal energy $\epsilon$ are computed from $\rho, T$, and $Y_{e}$ using an EOS table (see Section 2.1 below). From these, one can compute the specific enthalpy $h=1+\epsilon+P / \rho$. From $v_{i}$, one can compute the Lorentz factor $W=\alpha u^{t}, \alpha$ being the lapse and $u^{t}$ being the time component of the 4-velocity. The hydrodynamic evolution equations take the form of conservation laws for a set of "conservative" variables. These include a density variable, $\rho_{*}=\sqrt{\gamma} W \rho$, an energy variable, $\tilde{\tau}=\rho_{*}(h W-1)-\sqrt{\gamma} P$, a momentum variable $\tilde{S}_{i}=\rho_{*} h u_{i}$, and a composition variable $\rho_{*} Y_{e}$, where $\gamma$ is the determinant of the 3-metric, and $u_{i}$ are the covariant components of the 4-velocity. Note that we do not include neutrino pressure or energy in the definitions of $\tilde{\tau}$ and $\tilde{S}_{i}$; the neutrino radiation field appears only through source terms in the evolution equations (see Section 2.3).

To carry out a timestep, we begin by computing the conservative variable fluxes on cell faces using fifth-order WENO reconstruction (Liu et al. 1994; Jiang \& Shu 1996) of $\rho, T$, and $u_{i}$ and an HLL approximate Riemann solver (Harten 1983). From these cell boundary fluxes, the conservative variables are advanced forward in time. From the evolved conservative variables, the "primitive" variables $\rho, T$, and $v_{i}$ must then be recovered. This amounts to a two-dimensional root-finding problem to find the values of $W$ and $T$ that reproduce the evolved conservative variables. For some combinations of conservative variables, no corresponding set of primitive variables exists. For this reason, relativistic hydrodynamics codes often impose the condition $\tilde{S}^{2}<\tilde{\tau}\left(\tilde{\tau}+2 \rho_{*}\right)$ (Etienne et al. 2008). This inequality assumes $h \geqslant 1$ for all physical values of $\rho$ and $T$. One feature of nuclear-based EOSs is that the internal energy becomes negative at sufficiently low densities and temperatures. For an EOS with a specific enthalpy minimum of $h_{\min }$, the invertibility condition becomes

$$
\tilde{S}^{2}<\tilde{S}_{\max }^{2}=\tilde{\tau}\left(\tilde{\tau}+2 \rho_{*}\right)+\left(1-h_{\min }^{2}\right) .
$$

To avoid divisions by zero, we impose a density floor of $6 \times 10^{3} \mathrm{~g} \mathrm{~cm}^{-3}$. Gas near the surface of the matter, at points with densities more than a few decades below the maximum, cannot be expected to be evolved accurately; high temperatures and velocities tend to develop there. We therefore impose a maximum value on $T$ and $u^{2} \equiv \gamma^{j k} u_{j} u_{k}$ in the low-density region outside the star, tail, and disk (as in Foucart et al. 2013, but with modifications). The ceiling on $T$ is the minimum temperature in the EOS table at densities below $10^{-5} \rho^{\max }(t)$, where $\rho^{\max }(t)$ is the instantaneous maximum density. We smoothly taper this treatment to densities 10 times larger than the lower threshold by making the ceiling a linear function of density rising to $10 \mathrm{MeV}$. Above $10^{-4} \rho^{\max }(t)$ no temperature modification is applied. The ceiling on $u^{2}$ is 0 at densities below $10^{-7} \rho^{\max }(t)$, and is smoothly tapered to 1000 at densities 100 times larger than the lower threshold. Above $10^{-5} \rho^{\max }(t)$ no velocity modification is applied. In addition, these density thresholds are increased by a factor of 100 in a region very near the black hole. $Y_{e}$ evolution is not modified at any density. By running segments of the evolution with different density thresholds for the above treatments, we confirm that their main effect is to reduce noise in the neutrino luminosity during tidal disruption-noise that is dwarfed by the post-disruption signal anyway.

\subsection{Equation of State}

In this work we describe the fluid with the Lattimer \& Swesty (hereafter LS) EOS. This EOS is derived from a compressible liquid-drop model with a Skyrme nuclear force and includes contributions from free nucleons, alpha particles, and a single type of heavy nucleus (Lattimer \& Swesty 1991). We set the nuclear incompressibility parameter, $K_{0}$, to $220 \mathrm{MeV}$ and the symmetry energy $S_{v}$ to $29.3 \mathrm{MeV}$. Electrons, positrons, and photons are added using the routines of Timmes \& Arnett (1999).

We employ the LS EOS in our evolutions as a table of the following thermodynamic and composition quantities: internal energy; pressure; sound speed; neutron, proton, and electron chemical potentials; and neutron, proton, alpha particle and characteristic heavy nucleus mass fractions (along with the characteristic heavy nucleus's average mass and charge number). We store the table with the following ranges and resolutions: $\rho \in\left[10^{8}, 10^{16}\right] \mathrm{g} \mathrm{cm}^{-3}, N=250$, log spacing; $T \in[0.01,251] \mathrm{MeV}, N=120$, log spacing; $Y_{e} \in$ $[0.035,0.53], N=100$, linear spacing. As in O'Connor \& Ott (2010) we perform tri-linear interpolations for intermediate values. The original table and access routines are available at http://www.stellarcollapse.org. Continuous extrapolations of $P$ and $\epsilon$ outside the tabular bounds are defined for primitive variable recovery. Note, however, that if $T$ or $Y_{e}$ at a fluid grid point evolves to a value outside the table bounds, it is reset to the minimum or maximum. Thus, becaue of this resetting, the extrapolations used for these variables cannot influence the evolution. However $\rho$ is allowed to take any value above the floor density. For densities above the floor density and below the table minimum, we set the (negligible) pressure using a polytropic law with constants chosen for each $T$ and $Y_{e}$ to smoothly match the low-density bound of the table. Densities greater than those covered by the LS table do not occur in our simulation.

Just below nuclear saturation density a phase transition occurs between nuclei at low densities and uniform nuclear matter at high densities. The LS EOS captures this transition smoothly in free energy, but other parameters suffer from jumps between the two phases. During the inversion from conservative to primitive variables, we solve for temperature an equation involving enthalpy and pressure. The inversion, therefore, is 
difficult when either $h(T)$ or $P(T)$ are not smooth. We find that the sharp transition is most disruptive to inversion at densities near $\rho \sim 10^{14} \mathrm{~g} \mathrm{~cm}^{-3}$, and temperatures near $T \sim 1 \mathrm{MeV}$. We resolve the issue by "polishing" the table in the temperature dimension, applying a gaussian smoothing kernel of width $0.05 \mathrm{MeV}$ to the stored values of internal energy and pressure.

\subsection{Initial Data}

We choose initial parameters that we expect to yield a massive accretion disk, and high accretion efficiencies, giving us an upper bound on energetics. The binary is characterized by a low-mass ratio of $q=4$, a high prograde aligned spin of $a^{*}=0.9$, and an initial orbital separation yielding 6 orbits of inspiral before disruption. In addition, we set the star's initial temperature to $0.01 \mathrm{MeV}$, and choose a $Y_{e}$ profile that enforces $\beta$-equilibrium with zero neutrino chemical potentials $\left(\mu_{v_{i}}=0\right)$. The star's baryonic rest mass is $1.55 M_{\odot}$. In isolation, it would have a gravitational mass of $1.40 M_{\odot}$ and an areal radius of $12.7 \mathrm{~km}$.

To compute initial data we solve the extended conformal thin sandwich equations using multi-domain pseudospectral methods, as detailed in Foucart et al. (2008), using the Spectral Elliptic Solver (Pfeiffer 2003; Pfeiffer et al. 2003, 2005). The numerical domain is decomposed into a set of touching but not overlapping "subdomains," comprised of spherical shells, filled spheres, cylinders, and distorted cubes, arranged to reflect the symmetries in the configuration. We use the same method to represent the metric in evolutions (see e.g., Figures 1 and 2 in Foucart et al. 2013). One difficulty arising within a spectral framework is the spurious Gibbs oscillations that can occur at discontinuities, for example at the surface of the star. Thus, a critical technique in our method involves capturing the surface at a subdomain boundary, where the abutting spectral domains have no difficulty representing the non-smooth field. For the polytropes used in our previous work (Duez et al. 2010; Foucart et al. 2011, 2012, 2013; Foucart 2012) the density profile is smooth and easy to resolve. However new difficulties present themselves with the complexity of the stellar structure derived from the LS EOS.

Polytropes have density profiles that fall off as $\rho \propto\left(r-r_{*}\right)^{n}$ close to the surface (Gundlach \& Please 2009), where $r_{*}$ is the stellar radius, and $n$ is related to the polytropic index by $\Gamma \equiv 1+1 / n$. Thus, density profiles of $\Gamma=2$ polytropes behave linearly at the surface. To understand the effect of realistic EOSs on stellar surfaces we may use the effective adiabatic index, defined $\tilde{\Gamma} \equiv(d \log P / d \log \rho)_{S}$. The LS EOS, at low temperature and $Y_{e}$ in $\mu_{v_{i}}=0 \beta$-equilibrium, is extremely stiff near the central density of a $1.4 M_{\odot}$ star, with $\tilde{\Gamma} \sim 7 / 2$. It begins to soften dramatically at $\sim 10^{14} \mathrm{~g} \mathrm{~cm}^{-3}$, to $\tilde{\Gamma} \sim 1 / 2$. At even lower densities $\tilde{\Gamma}$ asymptotically approaches the adiabatic index of a relativistic Fermi gas, $\tilde{\Gamma} \sim 4 / 3$. These transitions occur very near the stellar surface, at $r \gtrsim 0.95 r_{*}$ in our evolution coordinates. The dramatic change in $\tilde{\Gamma}$ presents a kink in the density profile that is difficult to resolve, especially with spectral methods. Because the kink occurs over a range of radii, it cannot be captured by a subdomain boundary, as the well-defined surface of a polytrope can.

We can often improve the performance of our initial data solves by manipulating the EOS minimally. We find we can bypass most of the non-smoothness of the stellar profile by appending a simple polytrope to the low-density portion of the cold EOS. This makes the initial star more amenable to spectral representation. Specifically, we use $P(\rho)=\kappa_{0} \rho^{\Gamma_{0}}$ to describe pressures and

$$
\epsilon(\rho)=\frac{\kappa_{0}}{\Gamma_{0}-1} \rho^{\Gamma_{0}-1}+\epsilon_{\text {shift }}
$$

to describe energies below $\rho_{\text {break }}$. $\rho_{\text {break }}$ is determined by enforcing continuity in the effective adiabatic index $\left(\tilde{\Gamma}\left(\rho_{\text {break }}\right)=\right.$ $\left.\Gamma_{0}\right), \kappa_{0}$ by enforcing continuity in pressure, and $\epsilon_{\text {shift }}$ by enforcing continuity in energy. We use $\Gamma_{0}=2$ with the LS EOS at $T=0.01 \mathrm{MeV}$ and $Y_{e}$ in $\mu_{v_{i}}=0 \beta$-equilibrium. This implies $\rho_{\text {break }}=4.49 \times 10^{13} \mathrm{~g} \mathrm{~cm}^{-3}, \kappa_{0}=1.40 \times 10^{4} \mathrm{~g}^{-1} \mathrm{~cm}^{5} \mathrm{~s}^{-2}$, and $\epsilon_{\text {shift }}=9.13 \times 10^{17} \mathrm{erg} \mathrm{g}^{-1}$.

The effects of this manipulation on the bulk of the star are minimal. It is helpful to bear in mind that the central density of a $1.4 M_{\odot}$ star described by this EOS is $\sim 7 \times 10^{14} \mathrm{~g} \mathrm{~cm}^{-3}$; we append the polytrope more than an order of magnitude below this density. Though the star's radius decreases by several percent, its rest mass changes by one part in $10^{5}$. Test evolutions of isolated stars show that perturbations from equilibrium, due to switching from the modified EOS used in the initial data to the true EOS used in the evolution, are smaller than the numerical noise at our typical resolutions.

In practice the manipulated density profile still falls off somewhat non-smoothly at the surface, and we can sometimes gain further accuracy in our initial data by adding an additional subdomain, isolating the outer $20 \%$ of the star (in radius) to a thin spherical shell.

Note that we limit these two surface-capturing methods to the initial data solve, during which the metric and fluid variables are represented on spectral subdomains. During the evolution, the fluid variables are represented on a finite-difference grid, where we also employ a high-resolution shock-capturing technique that was designed to describe fluid discontinuities.

Because the quasi-equilibrium approximation ignores the infall motion that should be present initially, using this data directly results in eccentric orbits, which could affect the disk and outflow masses. Therefore, we perform one iteration of eccentricity reduction (see Pfeiffer et al. 2007 for details) to incorporate the initial infall and to reduce the initial eccentricity to $\sim 0.01$.

\subsection{Neutrino Leakage Scheme}

Neutrino leakage schemes attempt to account for the energy loss and electron number alteration caused by the emission of neutrinos by the nuclear fluid. To accomplish this, one first estimates the local effective energy emission rate $Q_{v}$ $\left(\mathrm{erg} \mathrm{s}^{-1} \mathrm{~cm}^{-3}\right)$, the effective lepton number emission rate $R_{v}$ $\left(\mathrm{s}^{-1} \mathrm{~cm}^{-3}\right)$, and the neutrino radiation pressure $P_{\nu}$ as measured in a local Lorentz frame comoving with the fluid. In such a frame

$$
\begin{gathered}
\partial_{t}\left(\rho Y_{e}\right)=-R_{v} m_{U}, \\
\partial_{t} T^{00}=-Q_{v}, \\
\partial_{t} T_{j 0}=-\partial_{j} P_{v},
\end{gathered}
$$

where $m_{U}$ is the atomic mass unit, and $T^{\alpha \beta}$ is the stress tensor. Then the energy, momentum, and composition source terms follow directly from putting the above in generally covariant form. In the comoving Lorentz frame of Equations (2)-(4), $u^{0}=1, u^{j}=0$, and $g_{\alpha \beta}=\eta_{\alpha \beta}$, so a covariant form of the neutrino sources is

$$
\nabla_{\alpha}\left(\rho Y_{e} u^{\alpha}\right)=-R_{\nu} U
$$




$$
\nabla_{\alpha} T^{\alpha \beta}=-Q_{\nu} u^{\beta}-\left(g^{\alpha \beta}+u^{\alpha} u^{\beta}\right) \nabla_{\alpha} P_{\nu} .
$$

For the time derivative of $P_{v}$, we assume simple advection $u^{\alpha} \nabla_{\alpha} P_{v}=0$, which is not quite right, since the neutrino field changes in ways other than advection, but the effect of the $\partial_{t} P_{v}$ source in our simulations (and, indeed, the effect of all neutrino pressure terms) is quite small, so a more accurate estimate is not necessary.

For the computation of $Q_{v}$ and $R_{v}$, our code is essentially an extension of the GR1D neutrino leakage code (O'Connor $\&$ Ott 2010) to three dimensions. That code in turn closely follows the leakage schemes of Ruffert et al. (1996) and Rosswog \& Liebendörfer (2003). In optically thin regions, emission rates should be given by the local rates of neutrinogenerating interactions for each neutrino species $v_{i}: Q_{v_{i}}^{\text {free }}$ and $R_{v_{i}}^{\text {free }}$. In optically thick regions, energy and leptons escape via diffusion at rates $Q_{v_{i}}^{\text {diff }}$ and $R_{v_{i}}^{\text {diff }}$. The effective rates come from interpolating these; e.g., for energy emission,

$$
Q_{v_{i}}=\frac{Q_{v_{i}}^{\text {free }} Q_{v_{i}}^{\text {diff }}}{Q_{v_{i}}^{\text {free }}+Q_{v_{i}}^{\text {diff }}} .
$$

Like most other leakage schemes, we include three neutrino species, $v_{e}, \bar{v}_{e}$, and $v_{x}$, where $v_{x}$ includes $\mu$ and $\tau$ neutrinos and antineutrinos. Then $Q_{v}=Q_{v_{e}}+Q_{\bar{v}_{e}}+4 Q_{v_{x}}$ and $R_{v}=R_{v_{e}}-R_{\bar{v}_{e}}$.

For free emission rates, we include $\beta$-capture processes $\left(e^{-} p \rightarrow n v_{e}\right.$ and $\left.e^{+} n \rightarrow p \bar{v}_{e}\right)$, electron-positron pair annihilation, plasmon decay, and nucleon-nucleon Bremsstrahlung emission. (For the latter process, we use the rate given in Burrows et al. 2006.) In calculating the opacity, we include scattering on nucleons and heavy nuclei and absorption on nucleons. All particle species, including the neutrinos, are assumed to have Fermi-Dirac distribution functions.

As in GR1D, we assume the neutrino chemical potentials used to calculate the emission rates are

$$
\mu_{v_{i}}=\mu_{v_{i}}^{\mathrm{eq}}\left(1-e^{-\left\langle\tau_{v_{i}}\right\rangle}\right),
$$

where $\mu_{v_{i}}^{\text {eq }}$ is the $\beta$-equilibrium value of $\mu_{v_{i}}\left(\mu_{v_{e}}^{\text {eq }}=-\mu_{\bar{v}_{e}}^{\text {eq }}=\right.$ $\left.\mu_{e}+\mu_{p}-\mu_{n}, \mu_{v_{x}}^{\mathrm{eq}}=0\right)$ and $\left\langle\tau_{v_{i}}\right\rangle$ is the energy-averaged optical depth.

Given the primitive variables and neutrino chemical potentials, we can compute $\tau_{\nu_{i}}\left(E_{v_{i}}\right)$, the optical depth of neutrino species $v_{i}$ at energy $E_{v_{i}}$ along some path parameterized by $\ell$, with the line integral

$$
\tau_{v_{i}}\left(E_{v_{i}}\right)=\int\left[\lambda_{v_{i}}\left(E_{v_{i}}\right)\right]^{-1} d \ell=E_{v_{i}}^{2} \int \hat{\lambda}_{v_{i}}{ }^{-1} d \ell,
$$

where $\lambda_{v_{i}}\left(E_{v_{i}}\right) \equiv E_{v_{i}}{ }^{-2} \hat{\lambda}_{v_{i}}{ }^{-1}$ is the mean free path, and we have taken advantage of the fact that all the absorption and scattering cross-sections considered have a common neutrino energy dependence, $\sigma_{v_{i}} \propto E_{v_{i}}^{2}$, which can be factored out. Using the optical depth, the diffusion rates $Q_{v_{i}}^{\text {diff }}$ and $R_{v_{i}}^{\text {diff }}$ are estimated as in Rosswog \& Liebendörfer (2003).

The calculation of $\tau_{v_{i}}$ uses interaction cross-sections that depend on $\mu_{v_{i}}$, and by Equation (8), these themselves depend on $\left\langle\tau_{v_{i}}\right\rangle$. GR1D iterates Equation (8), but since optical depth calculations are more expensive in three dimensions, and the interpolation formula is itself somewhat arbitrary, we have experimented with introducing a second optical depth variable $\left\langle\tau_{v_{i}}\right\rangle_{\text {approx }}$ for use in Equation (8). We have tried
1. setting $\left\langle\tau_{v_{i}}\right\rangle_{\text {approx }}$ equal to the energy average of $\tau_{v_{i}}\left(E_{v_{i}}\right)$ and, as in GR1D, iterating to rough convergence,

2. limiting the code to one iteration per opacity calculation, starting from previous calculation's results for $\left\langle\tau_{\nu_{i}}\right\rangle_{\text {approx }}$ and $\mu_{v_{i}}$. Since the matter distribution changes little between opacity computations, this method is very similar to the first.

3. using an analytic fit for neutron stars:

$$
\log _{10}\left\langle\tau_{v_{i}}\right\rangle_{\text {approx }}=0.96\left(\log _{10} \rho_{\text {cgs }}-11.7\right)
$$

4. modifying the above to capture the effect of temperature on the average neutrino energy, and hence the cross-section:

$$
\log _{10}\left\langle\tau_{v_{i}}\right\rangle_{\text {approx }}=0.96\left(\log _{10} \rho_{\mathrm{cgs}}-11.7\right)\left(\frac{T}{0.1 \mathrm{MeV}}\right)^{2} \text {. }
$$

The effect of the assumption for $\left\langle\tau_{v_{i}}\right\rangle_{\text {approx }}$ on the luminosity is fairly small for all epochs of our simulation (of order $10 \%$ or less), and the runs below use the simplest scheme (3). The effect on the lepton number emission rate can be tens of percents at times, meaning the choice of neutrino chemical potential may have some effect on the composition evolution of the disk, an issue we intend to explore more deeply in future work. As a first attempt to gauge the importance of this choice, we have carried out numerical experiments evolving our postmerger accretion disk at one resolution using different $\left\langle\tau_{v_{i}}\right\rangle_{\text {approx }}$ assumptions, namely schemes 2 and 3 . Switching from scheme 3 to 2 does produce transient behavior, during which time $R_{v}$ drops significantly, as points near the neutrinosphere adjust to the different opacity profile. After a short time, $R_{v}$ recovers, and the subsequent evolutions track each other decently. The notable aspect of the disk $Y_{e}$ evolution, the deleptonization at late times as the disk cools, is found in both schemes and does not seem to be an artifact of the chemical potential choice. It should be emphasized that $\left\langle\tau_{v_{i}}\right\rangle_{\text {approx }}$ is only used for Equation (8). The diffusion rates are determined using $\tau_{\nu_{i}}$ as computed in Equation (9).

The most costly part of the leakage scheme is the estimate of the optical depth. To compute this, we first interpolate the opacity (factoring out the $E_{v}^{2}$ neutrino energy dependence, as discussed above) onto a lower resolution three-dimensional grid. Then we compute line integrals of this quantity in seven directions to the computational boundary: one integral forward and backward on each coordinate axis, excluding $-z$ because of our grid's reflection symmetry, plus two diagonals based on the "most promising" of the coordinate directions (see Figure 1). The minimum line integral gives the optical depth. (More precisely, the optical depth is $E_{v}^{2}$ times this integral.)

To test our implementation of the leakage scheme, we have constructed spherically symmetric configurations of hot gas and compared our results ( $R_{v_{i}}, Q_{v_{i}}$, and the optical depth) with those of GR1D. We set the neutrino chemical potentials in the same way for this test to have a clean comparison. We tried spheres with a range of central density, temperature, and $Y_{e}$, so that some were optically thin and others optically thick. As expected, the two codes agreed.

The emission rates also allow us to compute the total neutrino energy and number luminosity radiated to infinity by performing proper integrals over $Q_{v}$ and $R_{v}$. For the energy luminosity at $r \rightarrow \infty, L_{v}$, we multiply the integrand by a redshift factor $g_{00}$ : one factor of $\sqrt{g_{00}}$ for the time dilation and one factor for the energy redshift. For the integral of $R_{v}$, we multiply by a redshift factor of $\sqrt{g_{00}}$. 


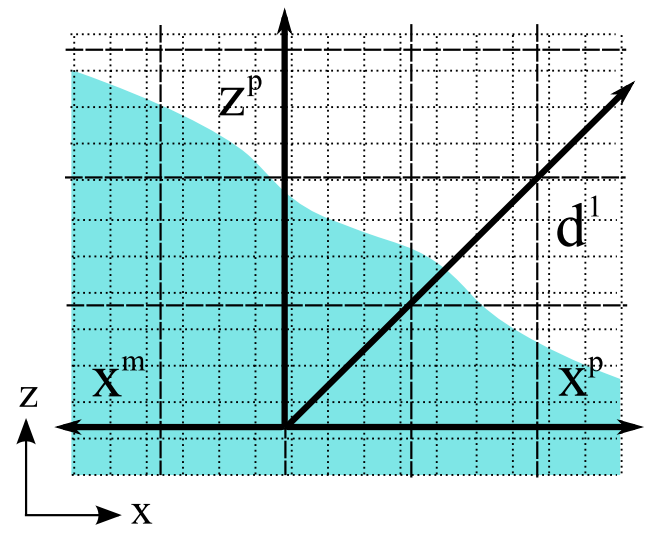

Figure 1. Schematic of the optical depth calculation. The fine (dotted) grid represents the finite-difference grid for the fluid evolution; the coarse (dashed) grid represents the opacity grid for the optical depth calculation. The blue region in the lower left represents dense fluid in which the neutrino mean free path is small. For each zone of the opacity grid, we first integrate five axial rays, excluding the $-z$ axis because of equatorial symmetry; three of these are labeled: $x^{m}, x^{p}, z_{p}$. Then we integrate two additional "most promising" diagonal rays, lying between the minimum optical depth axis and the two next-to-minimum axes; one of these is labeled: $d^{1}$.

(A color version of this figure is available in the online journal.)

\section{SUMMARY OF EVOLUTION: GLOBAL MEASURES AND CONVERGENCE}

Our evolution begins with a coordinate separation of $83 \mathrm{~km}$ between the centers of the two compact objects. The inspiral takes place over six orbits $(28 \mathrm{~ms})$ and is followed by tidal disruption and merger. We continue to evolve the spacetime and fluid $\sim 7 \mathrm{~ms}$ past merger. We define "merger" as the time when $50 \%$ of the matter has depleted, mainly by accretion (at this time, only a small fraction $\left(<10^{-3}\right)$ has fallen off the outer boundary). By $7 \mathrm{~ms}$ the metric is mostly stationary, but the accretion disk remains highly dynamic. The interpolation and communication of the fluid variables to the spacetime (pseudospectral) grid is the dominant computational cost during this phase of the evolution, so continuing to evolve the complete system for many tens of milliseconds would be prohibitive with the current code. Instead, we continue the evolution some $40 \mathrm{~ms}$ longer in the Cowling approximation, that is we evolve the fluid but leave the spacetime fixed.

The Cowling approximation ignores several effects. First, it neglects changes in the disk's and the black hole's gravitational pull. The mass accreted onto the black hole is some two orders of magnitude smaller than the black hole mass, so this effect is probably unimportant. Second, it neglects changes in the black hole's spin. We estimate the change in the Kerr spin parameter through the $40 \mathrm{~ms}$ of Cowling evolution by measuring the change in the angular momentum and mass of the fluid. If we were to continue evolving the metric, $a^{*}$ would decay (from $\sim 0.9$ ) by $2 \%$, pushing out the radius of the innermost stable circular orbit by less than 10\% (Bardeen et al. 1972). We do not expect this effect to play a significant role in the disk evolution. Third, the Cowling approximation cannot capture instabilities due to the disk's self-gravity. However, this should not qualitatively alter perturbations in the bulk of the disk. The threshold for gravitational instability can be estimated from Toomre's criterion: $Q_{T}=\kappa c_{s} /(\pi G \Sigma)>1$ for stability (Safronov 1960; Toomre 1964), where $c_{s}$ is the sound speed of the disk, $\kappa$ is the epicyclic frequency, $\Sigma$ is surface density, and $G$ is the gravitational constant. For the
Table 1

Summary of Numerical Resolutions

\begin{tabular}{lcccc}
\hline \hline & & L1 & L2 & L3 \\
\hline$N$ gridpoints & Spectral domain & $70^{3}$ & $75^{3}$ & $87^{3}$ \\
& Hydro domain & $120^{3}$ & $140^{3}$ & $160^{3}$ \\
$\langle d x\rangle(\mathrm{km})$ & Early: star & 0.21 & 0.18 & 0.16 \\
& Late: inner disk (vert/rad) & $0.9 / 2.7$ & $0.8 / 2.3$ & $0.7 / 2$ \\
\hline
\end{tabular}

Notes. $N$ is the total number of gridpoints in the evolution domains. $\langle d x\rangle$ is the average grid spacing on the hydro domain, calculated in evolution coordinates.

disk considered here, the minimum Toomre parameter there is about 20 except for the inner and outer edges. The small amount of matter in the outer regions of the grid is not yet in circular orbit equilibrium, so the stability condition is inapplicable. At the innermost region of the disk, $\kappa \rightarrow 0$ at the innermost stable circular orbit, but here the Cowling approximation does capture the main orbital instability. Fourth, the Cowling approximation discards the gravitational waves caused by the disk's nonaxisymmetry. For the observed mass quadrupole variations induced in the disk, gravitational waves will affect the modes on a timescale $E_{\text {mode }} / L_{\mathrm{GW}} \sim 10^{2} \mathrm{~s}$ (where $E_{\text {mode }} \sim M_{0} v^{2}(\delta \Sigma / \Sigma)^{2}$ is a characteristic energy of the spiral waves, and $L_{\mathrm{GW}}$ is the gravitational wave luminosity). Thus we may safely ignore this effect. Finally, this approximation "freezes in" any nonaxisymmetric modes present in the metric when we transition to the Cowling evolution. We see that the amplitude (normalized to the $m=0$ mode) of the $m \geqslant 1$ modes in the lapse are below $\sim 10^{-3}$ at all radii. We expect this nonaxisymmetry to feed back into the fluid at the same order of magnitude.

To check the accuracy and robustness of our results, we evolve the merger at 3 resolutions, which we label "L1," "L2," and "L3." See Table 1 for a summary of the resolutions. Since the spectral evolution uses adaptive meshing, the number of colocation points changes significantly over the course of an evolution, so we report average resolutions. The actual numbers of stored fluid grid points are half those shown in the table, since each stored number gives the fluid variables at a point below and a point above the equator, following our assumption of equatorial reflection symmetry. After tidal disruption, the coordinate system of our fluid grid is driven nonuniformly to higher resolution close to the black hole. Our high resolution corresponds to a fluid grid spacing of $\sim 160 \mathrm{~m}$ during inspiral and about $0.7 \mathrm{~km}$ vertical $/ 2 \mathrm{~km}$ radial in the inner disk after merger.

In addition to the convergence tests, we perform one merger, at the $\mathrm{L} 1$ resolution, without neutrino leakage. We label this run "L1nov." Comparing L1nov to L1 lets us isolate the effects of the neutrinos on the fluid.

In Figures 2 and 3, we show the evolution of several main global quantities for all runs. Comparing resolutions allows us to discern which evolution features are robust and which are numerical artifacts. For example, the neutrino luminosity $\left(L_{\nu}\right)$ spikes $2 \mathrm{~ms}$ after merger, but this feature appears to converge away as resolution is increased. Volume renderings of the fluid (see an equatorial slice in Figure 4) show that at these times, some of the nuclear matter is spread in a thin tidal tail which is underresolved at low resolutions. The luminosity at later times comes from the much better-resolved disk, and we see good agreement between the higher resolutions on $L_{v}$ after about $5 \mathrm{~ms}$. Figure 2 reveals that the rest mass of 


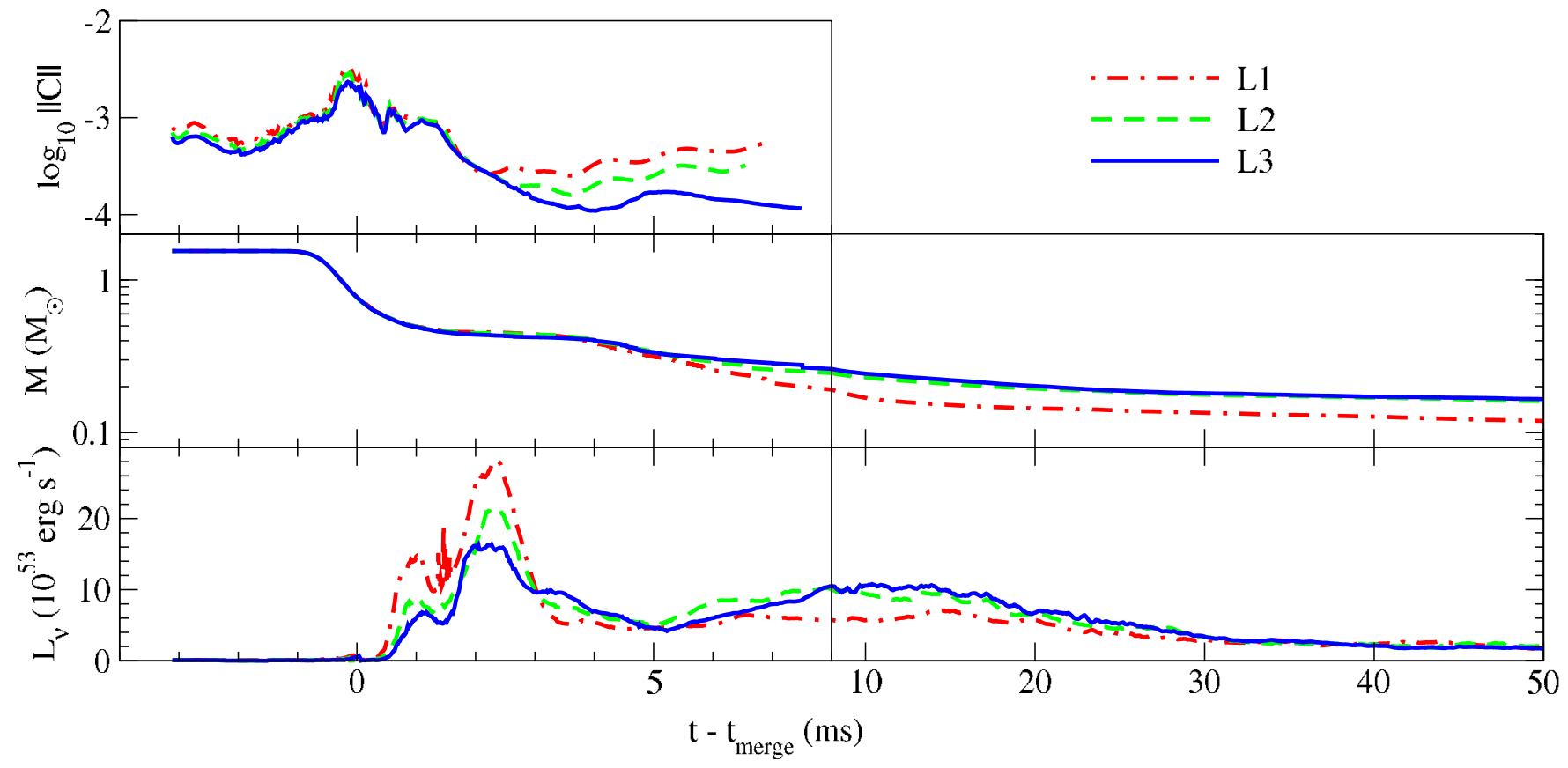

Figure 2. Global convergence measures, showing the three resolutions with leakage. $t_{\text {merge }}$ is the time at which $50 \%$ of the rest mass has depleted. We use different scalings for the time-axis before and after $8 \mathrm{~ms}$ in order to highlight the initial disruption and accretion. Top panel: $L^{2}$ norm of global generalized harmonic constraint violations (see Lindblom et al. 2006). We stop tracking this measure at $\sim 7 \mathrm{~ms}$ when we freeze the spacetime metric and begin the Cowling evolution. Middle panel: baryonic rest mass remaining on the computational grid (in units of $M_{\odot}$ ). The initial decrease beginning at $-1 \mathrm{~ms}$ is driven by accretion onto the black hole. The second decrease beginning near $3 \mathrm{~ms}$ is due to matter falling off the outer boundary of the computational domain. The disk mass quoted throughout $\left(M_{0} \approx 0.3 M_{\odot}\right)$ is the gravitationally bound mass outside the $\mathrm{BH}$ at $5 \mathrm{~ms}$ in the $\mathrm{L} 3$ evolution, i.e., the mass on the grid minus the instantaneous estimate of the remaining unbound mass discussed in Section 4. Bottom panel: total neutrino luminosity (in units of $10^{53} \mathrm{erg} \mathrm{s}^{-1}$ ), as measured at $r \rightarrow \infty$.

(A color version of this figure is available in the online journal.)

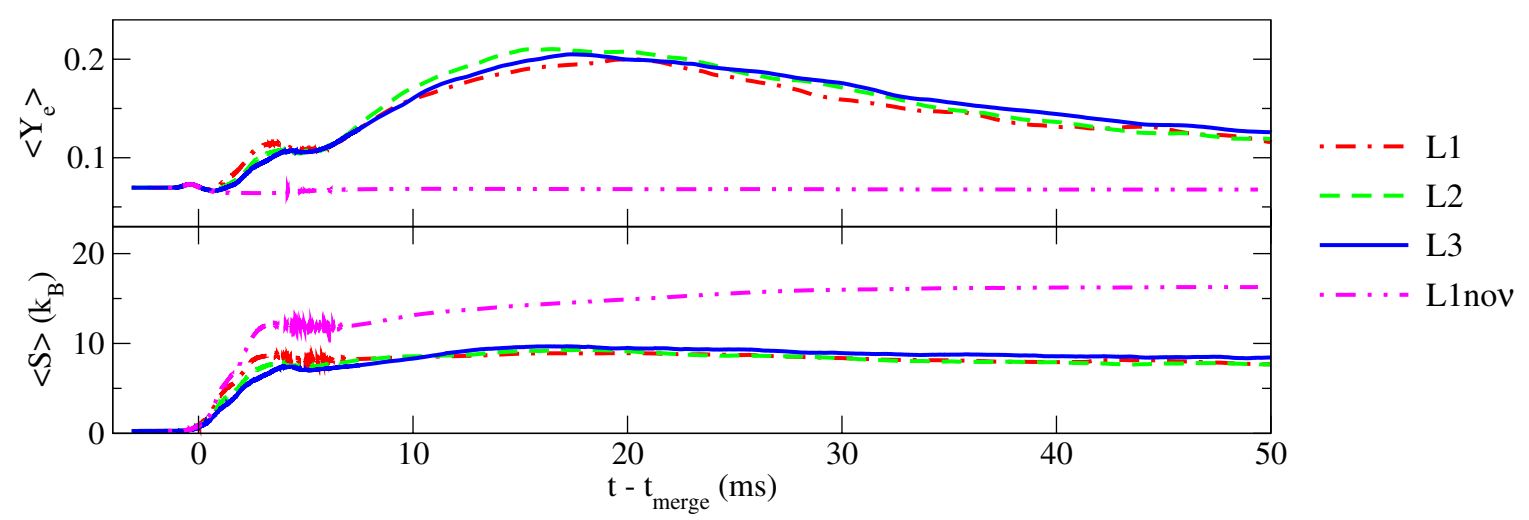

Figure 3. Secular chemical and thermal evolution of the disk for the three resolutions with leakage, and also L1nov. Top panel: mass-weighted average of the electron fraction. In the L1nov evolution, $Y_{e}$ simply advects with the flow. The early decreases at $0 \mathrm{~ms}$ and $3 \mathrm{~ms}$ are due to the major mass loss episodes: initial accretion, and loss of the tidal tail from the outer boundary. Bottom panel: mass-weighted average of the entropy, in units of $k_{B}$ baryon $^{-1}$.

(A color version of this figure is available in the online journal.)

L1 at late times differs from that of the higher resolutions significantly more than the high resolutions differ between themselves. By $10 \mathrm{~ms}$ this difference has settled to about $30 \%$. The reason for this is that resolution L1 generates a hotter, fatter, lower-density disk. The puffed-up disk drives more of its matter off of our computational domain, an effect that we can already see in outflow measurements by $2 \mathrm{~ms}$ after merger (see Section 4). Finally, in Figure 3, we again see that the different resolutions agree well on the post-merger composition and entropy, giving us confidence that these are roughly correct, though strict convergence is lacking. Given the strong shocks and turbulent-like behavior in the disk, this is unsurprising. However, comparing the leakage runs to L1nov, we see that the secular neutrino effects are much larger than the differences between resolutions.

\section{TIDAL DISRUPTION AND EJECTA}

In a BHNS merger, matter expelled at high velocity may ultimately become unbound from the central gravitational potential. In addition to enriching the interstellar medium with $r$-process elements (Lattimer \& Schramm 1974; Freiburghaus et al. 1999; Arnould et al. 2007; Korobkin et al. 2012), this nuclear matter may emit an electromagnetic signal. The radioactive nuclei formed in the neutron-rich fluid quickly fission, emitting high-energy beta- and gamma-radiation. This heats the ejecta, which becomes optically thin on an expansion timescale (days to weeks), and radiates thermalized photons, producing an isotropic kilonova (or "macronova," see Li \& Paczynski 1998; Roberts et al. 2011; Metzger \& Berger 2012; Rosswog 2013; Piran et al. 2013; Kasen et al. 2013; Nissanke et al. 2013) at 

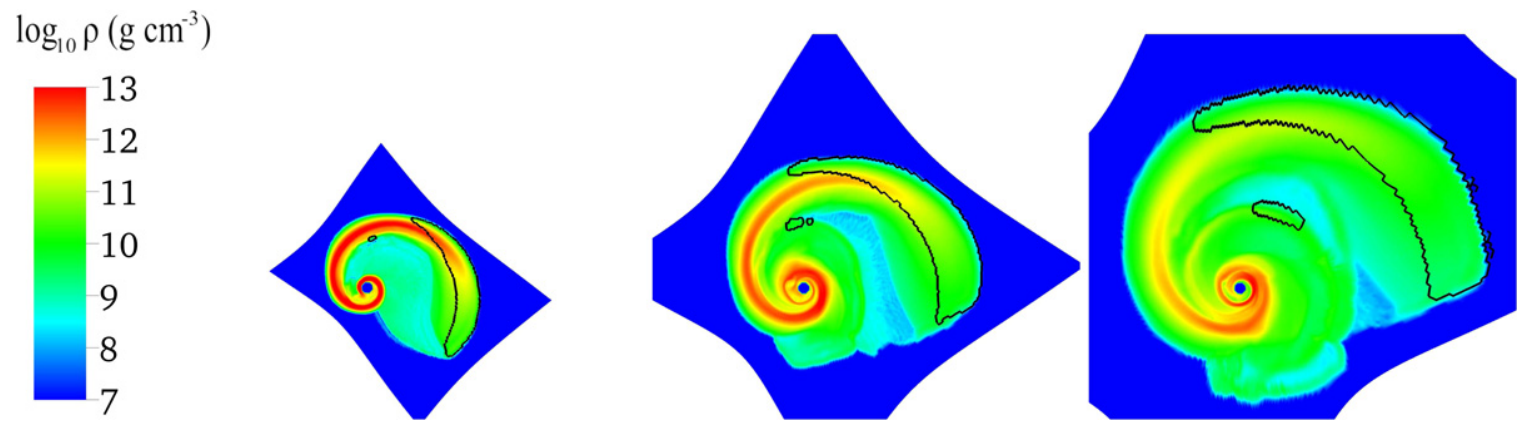

Figure 4. Equatorial slices of rest density from L 3 during disruption and tidal tail formation at 1, 2, and $3 \mathrm{~ms}$ after merger (from left to right). The unbound material is outlined in black. The field of view is fixed in all panels, so that each has a scale of approximately $680 \times 680 \mathrm{~km}^{2}$. The computational grid expands with the tail until it covers a circle of radius $\sim 400 \mathrm{~km}$ (in our asymptotically flat evolution coordinates) at which we remove matter from the grid. Note that we distort the coordinate map between the fundamental evolution frame and the frame in which the finite-difference grid is uniform in order to concentrate resolution near the black hole (see Duez et al. 2010).

(A color version of this figure is available in the online journal.)

optical, or perhaps infrared, wavelengths, depending on the highly uncertain opacity properties of the material (Kasen et al. 2013).

Additionally, the high-velocity ejecta form a blast wave in the circumbinary medium. At the shock front, magnetic fields amplify and accelerate electrons and positrons, thus emitting synchrotron radiation. This radio emission continues for a deceleration timescale (months to years) dependent upon the density of the surrounding medium (Metzger \& Berger 2012; Nakar \& Piran 2011; Piran et al. 2013; Nissanke et al. 2013).

These transient electromagnetic signals are of sufficient interest to warrant a thorough examination of any ejecta in our simulation. There are two dominant conveyers of ejecta in BHNS mergers: tidal tails and accretion disk winds. In this simulation, most of the unbound mass is produced by the tidal tail. A smaller amount is ejected in a plume during the early merger phase, as the infalling gas stream collides and shocks with itself. Also a small amount of outflow from the late disk is observed, but at low densities where numerical errors can introduce spurious acceleration and heating of the fluid. Note that this simulation ignores some effects that could drive strong winds (e.g., neutrino heating) and does not evolve long enough to see some others (e.g., large-scale He recombination). Below we review previous results, describe the formation of the tidal tail, describe our method of analyzing the ejecta, and finally report measures of its mass $\left(M_{\mathrm{ej}}\right)$, kinetic energy $\left(E_{\mathrm{ej}}\right)$, distribution of velocities, and composition $\left(\left\langle Y_{e}\right\rangle_{\mathrm{ej}}\right)$.

There are a number of recent studies examining ejecta from binary neutron star mergers (e.g., Rosswog 2013; Hotokezaka et al. 2013), high-eccentricity mergers (e.g., Stephens et al. 2011; East et al. 2012; East \& Pretorius 2012), and mergers including magnetic fields (e.g., Chawla et al. 2010). But here we survey results from low-eccentricity, nonmagnetized, BHNS studies to emphasize the influence of black hole spin. Lattimer \& Schramm (1974) made semianalytic estimates in a Schwarzschild spacetime. They found $M_{\mathrm{ej}} \sim 0$ to $0.14 M_{\odot}$ for stars that disrupt close to the black hole; no mass is ejected from stars that disrupt far away. Rosswog (2013) simulated two cases of $q \sim 4$ and $q \sim 7$ in a Newtonian potential. He found $M_{\mathrm{ej}} \lesssim 0.05 M_{\odot}$. Relativistic simulations have characteristically yielded more conservative ejecta estimates. Notably, high-mass ratio, compact neutron star, low black hole spin systems do not even disrupt. (See Pannarale et al. 2011 and Foucart 2012 for phenomenological models covering this parameter space.) In an excellent study focused on unique signatures from BHNS merger ejecta, Kyutoku et al. (2013) simulated a suite of tens of mergers with mass ratios of $q=3-7$ and prograde BH spins up to $a^{*}=+0.75$. They showed $M_{\mathrm{ej}} \sim 0.01 M_{\odot}-0.07 M_{\odot}$, with more matter ejected if the EOS is stiff. Recently, Foucart et al. (2013) simulated several mergers of $q=7$, with large $\mathrm{BH}$ spins of $\left|a^{*}\right|=0.9$ varying in inclination. In cases of aligned spin and orbital angular momentum, they found ejected masses of $M_{\mathrm{ej}} \sim 0.09 M_{\odot}$. Finally, in a study with nearly extremal BH spin, Lovelace et al. (2013) found $M_{\mathrm{ej}}$ could be as high as $0.3 M_{\odot}$. It appears, then, that for BHNS systems, one parameter region of interest for significant ejecta may be the parameter region of high spin.

In the present simulation, we see a large tidal tail form just before merger. About two orbits before merger $(t=-3 \mathrm{~ms})$ the coordinate separation of the two centers of mass has decayed to $40 \mathrm{~km}$ and the neutron star has become extremely distorted. After another revolution $(t=-1.5 \mathrm{~ms})$ the separation has decayed to $25 \mathrm{~km}$, the star overflows its Roche lobe, and it begins to accrete onto the black hole. A tidal tail has already formed from the trailing edge of the star, extending outward and lagging an orbit behind the core. At $t=0$, the core falls into the black hole. Over the next $3 \mathrm{~ms}$ the tidal tail sweeps out and away from the black hole (see Figure 4). We follow the tail by periodically resizing our computational domain outward to a radius of $\sim 400 \mathrm{~km}$, where we allow the tail to fall off of the grid. From its formation until its exit, the tidal tail is evolved on the computational domain for $\sim 5 \mathrm{~ms}$.

For a stationary spacetime, $u_{t}$ (the projection of the 4-velocity along the timelike Killing vector field) is a constant of motion for geodesics. Assuming the space is also asymptotically flat, $u_{t}$ gives the Lorentz factor $\left(\gamma=-u_{t}\right)$ of the particles as they escape to infinity. To the approximation that the spacetime is settled and that pressure is dynamically negligible, $u_{t}$ will be a constant along fluid parcels, and matter with $u_{t}<-1$ may be flagged as unbound. Neither approximation is strictly true, but both become better satisfied as the outflow expands outward away from the dynamical region and decompresses to low pressures. Thus, $u_{t}$ of fluid elements in the tail, and the total amount of unbound material in the tail, should become constant as the tail expands. If this settling happens before the tail leaves the computational grid, meaningful statements about the amount of outflow and its asymptotic velocity distribution can be made.

We integrate the mass satisfying the unboundedness condition $u_{t}<-1$ over times from $t \sim 0 \mathrm{~ms}$, just as the tidal tail is forming, to $t \sim 7 \mathrm{~ms}$, after the tail has entirely fallen off 


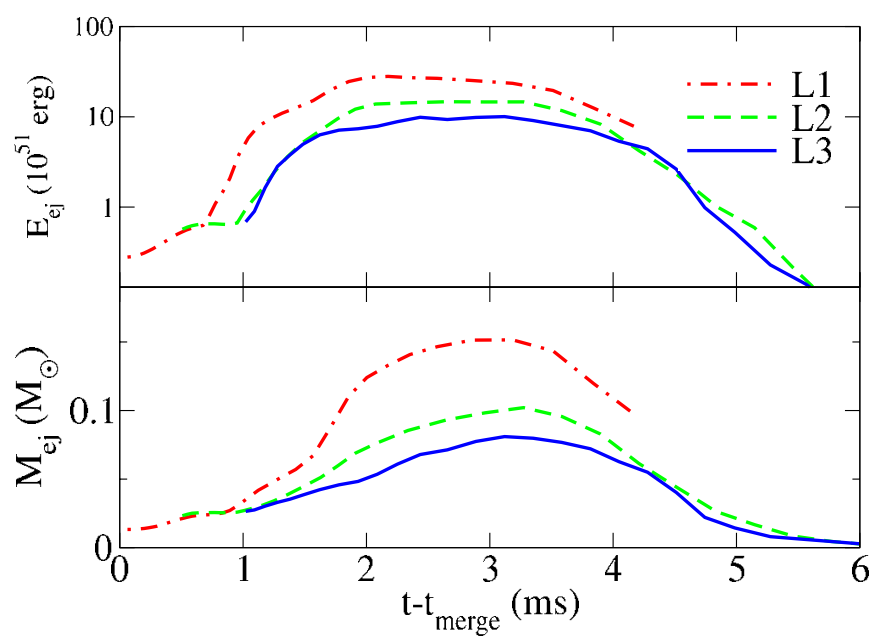

Figure 5. Integrals of the unbound fluid on the grid. Top panel: total asymptotic kinetic energy. Bottom panel: total rest mass. For both integrals a sphere of $73 \mathrm{~km}$ near the black hole was masked out. The drop-off after $3 \mathrm{~ms}$ is due to the loss of matter through the outer boundary.

(A color version of this figure is available in the online journal.)

the computational domain. We mask out regions within $50 M_{\odot}$ $(73 \mathrm{~km})$ of the $\mathrm{BH}$ in the evolution coordinates. At this distance the Newtonian gravitational potential is $M_{\mathrm{BH}} / r \lesssim 0.15$. The integral of total mass ejected is robust against $30 \%$ variations in the mask radius. Furthermore, our velocity cap (see Section 2 ) is only applied at densities below $\sim 10^{8} \mathrm{~g} \mathrm{~cm}^{-3}$ during this epoch, a full three orders of magnitude below the maximum density in the tail.

We check the validity of our assumption that the spacetime has become stationary by estimating the timescale that $u_{t}$ will change due to changes in the metric. For a nonrelativistic geodesic, the leading order term for the time derivative is $\dot{u}_{t} \approx g_{t t, t}$, so the timescale on which metric nonstationarity can produce a large relative change in energy is $\approx\left(-u_{t}-1\right) / \dot{u}_{t}$. This is $\sim 50 \mathrm{~ms}$ to $1 \mathrm{~s}$ for our ejecta when near the edge of the computational domain. On the other hand, the metric is rapidly settling as the gravitational wavetrain overtakes the ejecta, and $\dot{u}_{t}$ drops by an order of magnitude in a few ms. Thus, the ejecta energy is not expected to change by a large amount. However, tracking the ejecta for an extra few milliseconds would significantly reduce this source of error, an important consideration for future simulations.

Measures of $M_{\mathrm{ej}}$ and $E_{\mathrm{ej}}$ from the three resolutions are shown in Figure 5, where we have calculated the total integral over unbound matter on the grid. This raw measure increases early as a growing amount of material is flung out from the disk and exits the masked region. In addition to the tail, volume renderings of the unbound matter reveal the existence of a plume of outgoing matter ejected above the equatorial plane, of lower density than the tidal tail visible in Figure 4. This second outflow is produced during the merger, perhaps by the shock waves in the infalling matter. The most energetic matter in this plume begins to fall off the computational domain after $2.5 \mathrm{~ms}$, whereas the dense tidal tail begins to fall off after $3 \mathrm{~ms}$. Therefore, we use the peak values of mass and kinetic energy in Figure 5 as conservative estimates. We use Richardson extrapolation to derive uncertainties from the highest two resolutions, and we assume 2 nd order convergence, though Figure 5 indicates approximately 6th order covergence. This method gives $M_{\mathrm{ej}}=0.08 \pm 0.07 M_{\odot}$, and $E_{\mathrm{ej}}=10 \pm 17 \times$ $10^{51} \mathrm{erg}$. These errors are upper bounds which are probably large

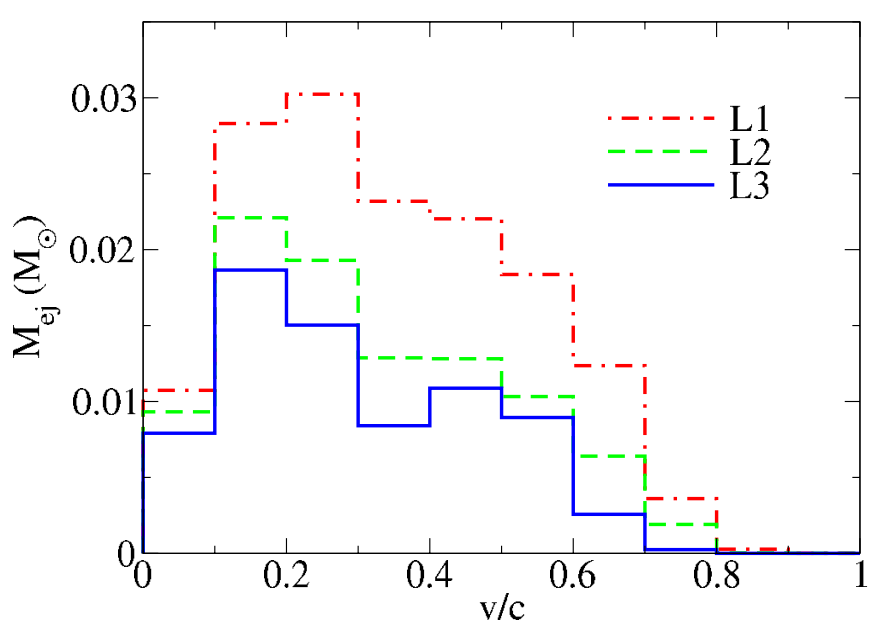

Figure 6. Unbound rest mass binned by magnitude of the asymptotic fluid velocity. We show L1, L2, and L3 at $\sim 2.7 \mathrm{~ms}$, just before the peak in Figure 5 . (A color version of this figure is available in the online journal.)

overestimates. Nonetheless, even at our highest resolution, the tail is poorly resolved: at $\sim 70 \mathrm{~km}$ wide, it is covered by $\sim 15$ grid cells. This is a common limit of grid-based methods; smoothedparticle hydrodyanmics methods neatly overcome this limit, but find their handicap in evolving accurate initial conditions for the tail.

The velocity distribution peaks across all resolutions at $0.2 c$, but the dispersion in velocities is large, with some matter escaping at $v>0.5 c$ (see Figure 6). Because average temperatures in the tidal tail are $\sim 1 \mathrm{MeV}$ as it leaves the computational domain, the fluid is still in nuclear statistical equilibrium, so our EOS is still valid. The material ejected from the system is neutron-rich, with average electron fraction $\left\langle Y_{e}\right\rangle_{\mathrm{ej}} \approx 0.1$.

\section{RELEVANT TIMESCALES FOR THE DISK}

The density in the accretion disk peaks at a circumferential radius $r$ of around $50 \mathrm{~km}$ from the black hole, and most of the disk mass is within $100 \mathrm{~km}$ of the black hole. At the density peak, the density is $\rho \approx 10^{12} \mathrm{~g} \mathrm{~cm}^{-3}$, the angular frequency $\Omega$ is around $3 \mathrm{kHz}$, and the sound speed $c_{s}$ is around $0.1 c$. The disk is hot and thick: $H / r \sim c_{s} /(\Omega r) \sim(1 / 3)$. The dynamical timescale of the disk is, to order of magnitude,

$$
T_{\mathrm{dyn}} \sim \Omega^{-1} \sim \frac{H}{c_{s}} \sim \frac{r}{c_{s}} \sim 1 \mathrm{~ms}
$$

Angular frequency decreases with distance from the black hole, so the orbital period at $r=120 \mathrm{~km}$ (roughly speaking, the disk's outer edge) is $10 \mathrm{~ms}$. Thus, even this outer region is evolved for several dynamical times. The perturbations in the inner disk rotate at frequencies similar to the local flow and thus orbit the black hole on millisecond periods.

The disk has strong entropy gradients in the inner and outer regions, so disk gas can experience buoyancy forces there on a timescale given by the Brunt-Väisälä frequency $N_{\mathrm{BV}}$,

$$
T_{\mathrm{S}} \sim\left|N_{\mathrm{BV}}\right|^{-1 / 2}=\left|-\rho^{-1}(\partial \rho / \partial S)_{P} g \cdot \nabla S\right|^{-1 / 2} \approx 1 \mathrm{~ms},
$$

where $g=\rho^{-1} \nabla P$ and $S$ is the specific entropy.

In addition to these dynamical processes, there are secular processes that alter the disk structure on longer timescales. One 
such effect is the depletion of baryonic mass in the disk. The disk's mass is $M_{0} \approx 0.3 M_{\odot}$ after merger (see Figure 2 ). For the first $30 \mathrm{~ms}$ after merger, accretion onto the black hole proceeds at a rapid rate of $\dot{M}_{0} \approx 2 M_{\odot} \mathrm{s}^{-1}$. During this time, the gas in the outer disk settles into circular orbit, which requires a transfer of angular momentum from the inner disk. (See Section 6 for a fuller discussion of the disk's angular momentum evolution.) The transport of angular momentum away from the inner disk by spiral density waves drives accretion onto the black hole. Eventually, the high-density middle region of the disk settles to equilibrium, but dynamical, nonaxisymmetric distortions persist near the disk's inner and outer edges (also discussed in Section 6). Spiral waves thus travel outward and drive a reduced rate of accretion throughout the simulation. After $30 \mathrm{~ms}$, the accretion rate has dropped by nearly an order of magnitude. At these late times, disk mass is also lost to a weak outflow from the inner disk at a comparable rate to the accretion into the black hole. Combining these effects, we can define a mass depletion timescale

$$
T_{\text {dep }} \sim \frac{M_{0}}{\dot{M}_{0}} \gtrsim 0.2 \mathrm{~s} .
$$

The disk's thermal evolution is driven by shock heating, compression, advection of heat into the black hole, and radiative cooling. The disk's thermal energy is defined as

$$
E_{\text {thermal }}=\int \rho_{*}\left[\epsilon-\epsilon_{\text {cold }}\left(\rho, Y_{e}\right)\right] d^{3} x,
$$

where $\epsilon$ and $\epsilon_{\text {cold }}\left(\rho, Y_{e}\right)$ are the actual specific internal energy and the specific internal energy at the lowest temperature in the EOS table, for which the gas is degenerate. We find $E_{\text {thermal }} \approx$ $10^{52} \mathrm{erg}$. The total neutrino luminosity is $L_{v} \sim 10^{53-54} \mathrm{erg} \mathrm{s}^{-1}$. Therefore, the cooling timescale due to neutrinos is

$$
T_{\text {cool }} \sim \frac{E_{\text {thermal }}}{L_{v}} \approx 10 \mathrm{~ms}-100 \mathrm{~ms} .
$$

It is important to remember that the disk is partly pressuresupported. Thus radiative energy loss may come from the potential energy reservoir via disk contraction, in addition to the more obvious thermal energy reservoir via disk cooling. Indeed we find that evolving without neutrino cooling leads to a disk that is not only hotter but also much more extended and less dense. The timescale for composition change is $N_{e} / R_{\nu}$, where $N_{e}$ is the number of electrons in the disk and $R_{v}$ is the total net lepton number change rate due to neutrinos. This is initially also about $10 \mathrm{~ms}$. Then, $20 \mathrm{~ms}$ after merger, balance between $v_{e}$ and $\bar{v}_{e}$ emission is roughly achieved, and the composition subsequently changes more slowly. Thus, the neutrino emission significantly influences the energy and composition of the disk over its lifetime.

\section{ACCRETION DISK: DYNAMICAL EQUILIBRIUM AND STABILITY}

\subsection{Disk Formation}

As can be seen from Figure 3, as the accretion stream collides with itself, shocks heat the gas for roughly one millisecond, until the density-averaged entropy settles at $\sim 8 k_{B}$ baryon $^{-1}$. A hot accretion disk forms in the vicinity of the black hole. In Figure 7, we show density snapshots of the disk at a representative time $\sim 30 \mathrm{~ms}$ later, and in Figure 8 , we show azimuthally averaged equatorial density as a function of circumferential
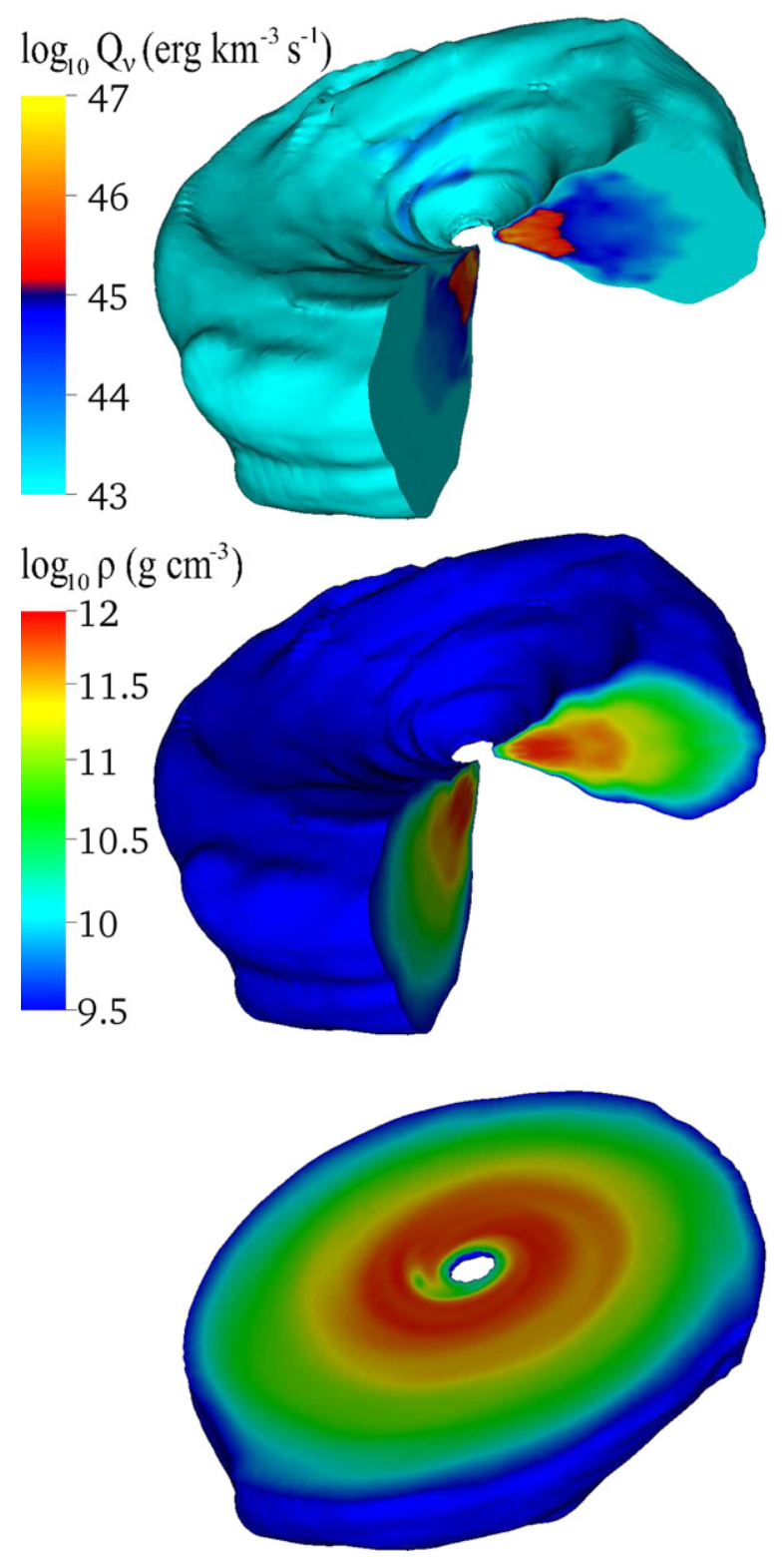

Figure 7. Three-dimensional distribution of neutrino energy loss and fluid density from L3, $30 \mathrm{~ms}$ after merger, depicted in evolution coordinates at a slanted view. Top panel: effective local neutrino power, $Q_{v}$ (no redshift applied), summed over all species (in units of $\mathrm{erg} \mathrm{km}^{-3} \mathrm{~s}^{-1}$ ). Middle and bottom panels: meridional and equatorial slices of density in the fluid rest frame. The equatorial slice reveals a spiral mode. In all three panels, densities below $10^{9.5} \mathrm{~g} \mathrm{~cm}^{-3}$ have been masked out to show the structure of the disk. The disk radius and halfthickness are $110 \mathrm{~km}$ and $45 \mathrm{~km}$, respectively.

(A color version of this figure is available in the online journal.)

radius. The maximum density remains at a fairly steady level of $\sim 10^{12} \mathrm{~g} \mathrm{~cm}^{-3}$. As shown in the bottom panel of Figure 8 , the densities and temperatures are sufficient to render the interior of the torus optically thick to all species of neutrinos, with optical depths (averaged over neutrino energy) of order 10.

The disk is initially extremely distorted and nonaxisymmetric. For a completely stable disk, one would expect the inner regions, where the dynamical timescale is shortest, to settle to a stationary axisymmetric state before the outer regions - as was seen, for example, in a recent BHNS $\Gamma$-law EOS merger carried out with the same code (Lovelace et al. 2013). (Unfortunately, we have not performed any $\Gamma$-law EOS merger simulations with similar mass ratio and black hole spin to the present case, so no proper 


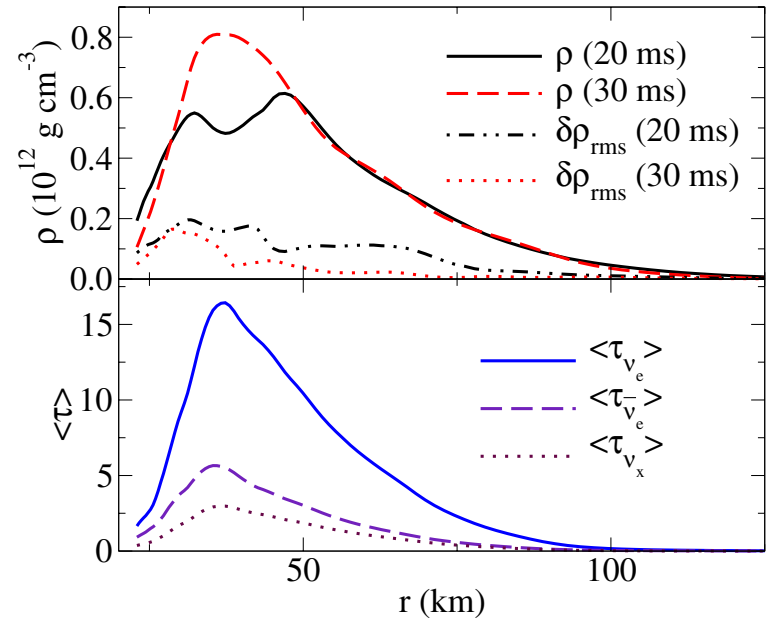

Figure 8. Top panel: azimuthally averaged profiles of the density $\rho$ together with its rms deviation from axisymmetry, $\delta \rho_{\mathrm{rms}} \equiv\left\langle(\rho-\langle\rho\rangle)^{2}\right\rangle^{1 / 2}$, plotted as functions of the circumferential radius at the equator $r$. Values for $20 \mathrm{~ms}$ and $40 \mathrm{~ms}$ after merger are shown. Bottom panel: density-weighted azimuthal and vertical average of the energy-averaged neutrino optical depth for each species of neutrino, computed $40 \mathrm{~ms}$ after merger.

(A color version of this figure is available in the online journal.)

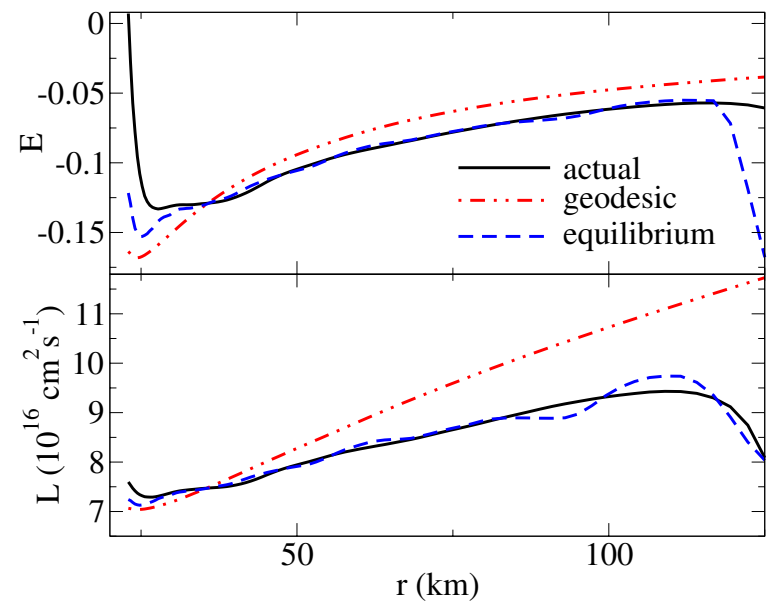

Figure 9. Energy and angular momentum of the disk $40 \mathrm{~ms}$ after merger. Top panel: azimuthally averaged equatorial orbital energy $E \equiv-u_{t}-1$. Bottom panel: azimuthally averaged angular momentum $L=-u_{\phi} / u_{t}$. In addition to $E$ and $L$ for the actual flow, we include values for circular orbit geodesic motion and circular orbit equilibrium (i.e., including pressure) motion.

(A color version of this figure is available in the online journal.)

comparison can be made.) We see instead that it is only the middle disk that settles to an approximately axisymmetric state after about $30 \mathrm{~ms}$. In the inner disk, very close to the black hole, clear, order unity deviations from axisymmetry in the form of trailing one-armed spirals persist throughout the evolution (see the bottom panel in Figure 7). These perturbations appear at the disk's inner edge, rotate at roughly the rate of the local fluid ( $\sim$ ms periods), expand outward and dissipate, and then reform many times during the disk evolution. Such behavior suggests that a fluid instability may be preventing the disk from promptly settling.

\subsection{Disk Equilibrium}

Further insight into the structure of the disk comes from the profiles of the specific orbital energy $\left(E \equiv-u_{t}-1\right)$ and angular momentum $\left(L=-u_{\phi} / u_{t}\right)$ shown in Figure 9. Each

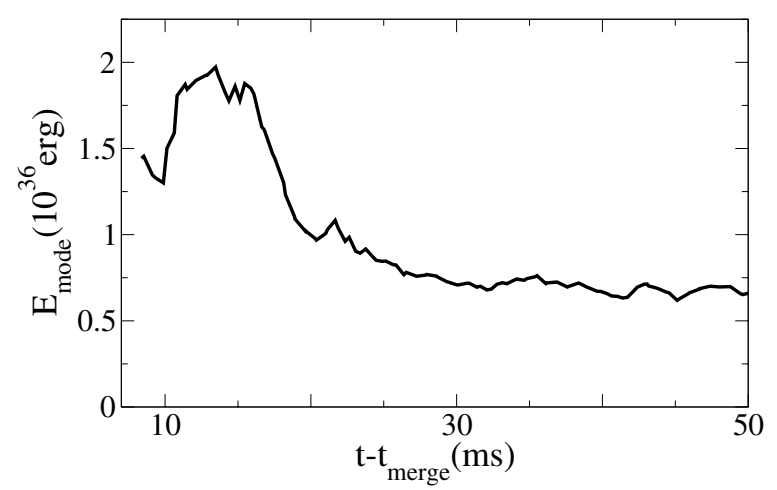

Figure 10. Deviation of the accretion disk from equilibrium, as measured by the difference between the actual and equilibrium orbital energy.

panel includes three curves. One is the actual $E$ or $L$ profile, measured on the equator and averaged over azimuthal angle. Another is the "Keplerian" $E$ and $L$, the values that would be found for geodesic equatorial circular orbit given the evolved spacetime metric of the system. The geodesic circular orbits are those for which $\nabla_{u} \boldsymbol{u}=0$. These curves thus include the effects of the disk's self-gravity (at least, as it was at the beginning of the Cowling evolution) but disregard pressure forces. We see that the angular momentum profile, for instance, is much shallower than would be found for an equilibrium thin (geodesic) disk. Finally, we include "equilibrium" curves, the $E$ and $L$ for equilibrium circular orbit given the existing metric, density, and pressure. These are orbits for which $\nabla_{\boldsymbol{u}} \boldsymbol{u}=-\nabla P /(\rho h)$. In regions where deviations from equilibrium are nonlinear, one must take care in identifying these curves with the true equilibrium about which the disk is perturbed, since $m=0$ perturbations in the pressure will feed back into the equilibrium condition. Given this qualification, we see that the highest-density regions do appear to be in equilibrium in an angle-averaged sense. The outer disk has sub-equilibrium angular momenta, so the gas remains in eccentric orbit. Interestingly, the energy and angular momentum increase dramatically at the inner edge of the disk. This feature is not present in the geodesic curves, but it is in the equilibrium curves, indicating that it is a consequence of a sharp pressure gradient near the inner edge of the disk. Below, we will consider the effect of these gradients on the expected stability of the disk. The high energies in the inner disk would make it easy to generate an outflow there. We do indeed observe some mass ejection from the inner disk, but at densities too low to be reliably modeled by our code.

The difference between the actual and equilibrium energy curves provides a measure of the mode energy, again, to the extent that equilibrium curves can reliably be computed from an incompletely settled pressure profile: $E_{\text {mode }} \equiv \int \rho_{*}(E-$ $\left.E_{\text {equilibrium }}\right) d^{3} x$. As shown in Figure 10, the mode energy is positive but decreases during the early, rapid accretion, phase. Then it settles and even shows episodes of growth, which may indicate that instabilities are stimulating these modes.

\subsection{Disk Stability}

In Figure 11, we plot the density-averaged pressure $P$, entropy $S$, temperature $T$, and electron fraction $Y_{e}$ as functions of circumferential radius-reducing from three dimensions by averaging these functions vertically and azimuthally. The entropy profile is fairly flat in the bulk of the disk, with the exception of a steep negative gradient $(d S / d r<0)$ in the hot inner region. Since in this region pressure increases 


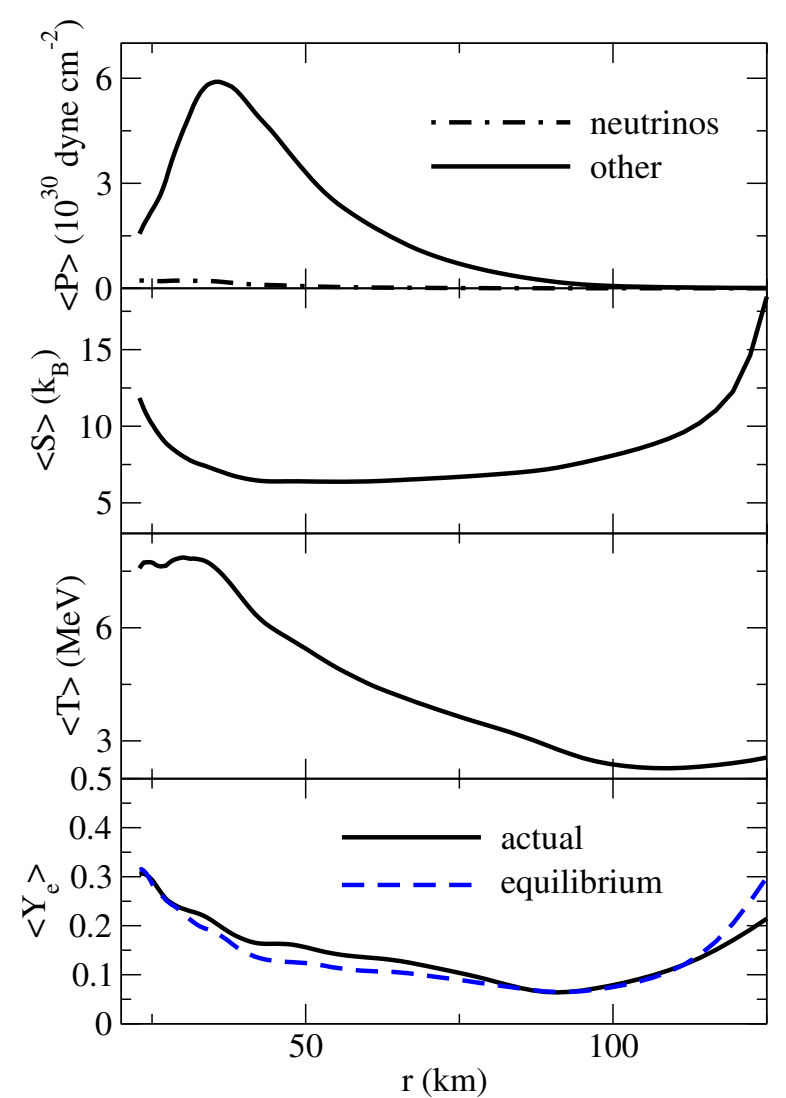

Figure 11. Profiles of the azimuthally and vertically averaged pressure, entropy, temperature, and electron fraction $40 \mathrm{~ms}$ after merger. For the pressure, we plot separately the contributions from the neutrinos and everything else (nucleons, electrons, photons). For $\left\langle Y_{e}\right\rangle$, we show both the actual value and the equilibrium value (at which $R_{v}=R_{v_{e}}-R_{\bar{v}_{e}}=0$ for the given density and temperature profile).

(A color version of this figure is available in the online journal.)

with radius, this entropy gradient is actually a stabilizing force. The $Y_{e}$ gradient also affects the buoyancy, but for these profiles, its effect is much smaller than that of the entropy. The relativistic Solberg-Høiland critera for convective stability of an axisymmetric equilibrium fluid are given, for a coordinate basis in which $g_{t r}=g_{r \phi}=0$, by (Seguin 1975)

$$
\begin{gathered}
\boldsymbol{\gamma} \cdot \nabla L+(\rho h)^{-2}(\partial \mathcal{U} / \partial S)_{P} \nabla P \cdot \nabla S \geqslant 0, \\
(\partial \mathcal{U} / \partial S)_{P}(\boldsymbol{\gamma} \times \nabla P) \cdot(\nabla S \times \nabla L) \geqslant 0,
\end{gathered}
$$

where $\mathcal{U}=\rho+\rho \epsilon$ is the total energy density and

$$
\boldsymbol{\gamma}=\left(u^{0} u_{0}\right)^{2}\left[\left(1-v^{2}\right)\left(g_{\phi \phi}\right)^{-1} u_{0}^{2} \nabla L-\nabla \Omega\right] .
$$

Equations (17) and (18) correspond to the familiar Newtonian radial and vertical stability conditions, respectively. The first term in Equation (17) dominates everywhere inside of $r \approx$ $120 \mathrm{~km}$, so $d L / d r$ determines the stability to radial convection through most of the disk. The condition for stability is met except in the inner edge $(r<27 \mathrm{~km})$ of the disk. This inner region should be unstable. The unstable gradient persists, and it is presumably connected to the inability of the inner disk to settle to stationary axisymmetry. The outer disk also has an unstable angular momentum gradient $(d L / d r<0)$, but it is stabilized by a strong positive entropy gradient. The entropy does not change much with height, but we do identify a negative gradient in

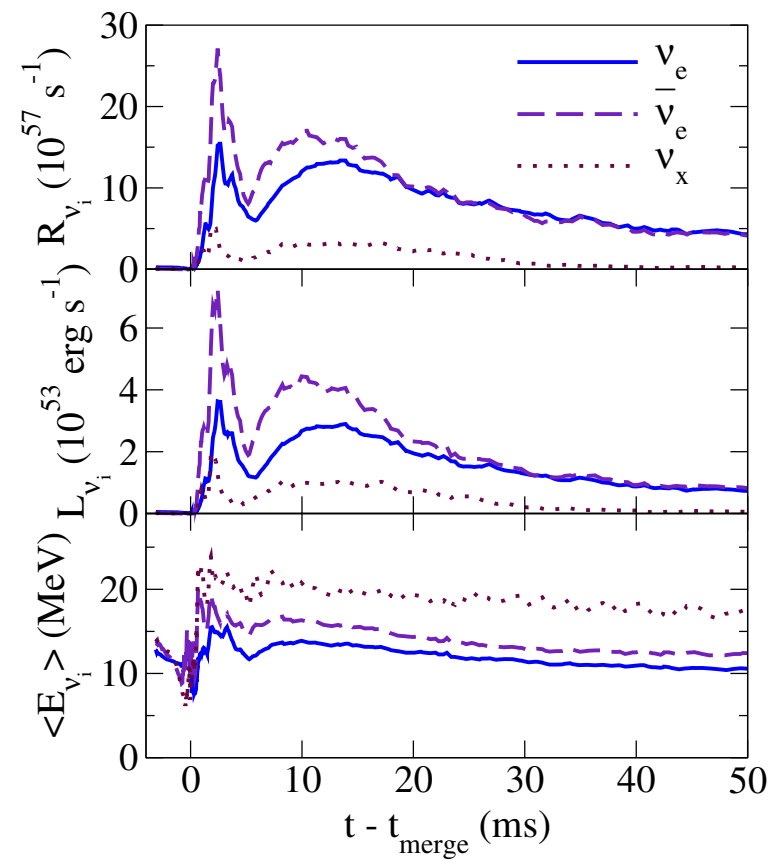

Figure 12. Neutrino emission characteristics by species. Top panel: lepton number luminosity, $R_{v_{i}}$ (in units of $10^{57} \mathrm{~s}^{-1}$ ). Middle panel: energy luminosity, $L_{v_{i}}$ (in units of $10^{53} \mathrm{erg} \mathrm{s}^{-1}$ ). The total luminosity is given by $L_{v_{e}}+L_{\overline{v_{e}}}+4 L_{v_{x}}$. $L_{v_{x}}$ is, thus, the luminosity of each individual $\mu$ and $\tau$ species. Bottom panel: average neutrino energy $\left\langle E_{v_{i}}\right\rangle$ (in unit of $\mathrm{MeV}$ ). All plots are taken from the L3 simulation, and are calculated for an observer at $r \rightarrow \infty$.

(A color version of this figure is available in the online journal.)

a region near the equator, leading to an instability according to Equation (18) there. Buoyant forces would be expected to correct this gradient on timescales $\left(-N_{\mathrm{BV}, \mathrm{z}}^{2}\right)^{-1 / 2} \sim 10 \mathrm{~ms}$, but apparently other processes (see Section 7 on processes that affect entropy evolution) are sufficient to keep the entropy profile roughly fixed.

In addition to local instability, the disk could be susceptible to global instabilities involving amplification of the unstable mode across its corotation radius, located at $r \approx 27 \mathrm{~km}$, given the mode's pattern speed (e.g., Papaloizou \& Pringle 1985).

The temperature is high $(\sim 8 \mathrm{MeV})$ in the inner disk, and in these regions, the contribution of trapped neutrinos to the total pressure is as high as $12 \%$. In the bulk of the disk, the neutrino pressure is negligible.

\section{NEUTRINO-DRIVEN DISK EVOLUTION}

\subsection{Disk Neutrino Luminosity}

Figure 12 breaks down the energy and number emission by neutrino species. Initially, $\bar{v}_{e}$ dominates over $v_{e}$ emission, as would be expected for a neutron-rich, proton-poor gas. As emission continues, protons become more numerous, and the neutrinosphere cools, so that positrons become less common than electrons. (The electrons are mildly degenerate, $\mu_{e} / k_{B} T \approx$ 1 , so the relative ratio of electron to positron density is quite sensitive to temperature.) Thus, $R_{v_{e}}$ and $R_{\bar{v}_{e}}$ become closer. At $20 \mathrm{~ms}$ after merger, these emission rates are sufficiently balanced that $Y_{e}$ thereafter evolves on the slightly longer timescale on which the disk itself is changing. In Figure 11, we compare the actual $Y_{e}$ to the equilibrium $Y_{e}$ profile, i.e., to the distribution $Y_{e}=Y_{e}^{\text {eq }}$ for the given $\rho$ and $T$, that would yield $R_{v}=R_{v_{e}}-R_{\bar{v}_{e}}=0$ everywhere in our leakage scheme. At early times, $Y_{e}<Y_{e}^{\text {eq }}$ for most of the matter and $Y_{e}$ grows. At late 


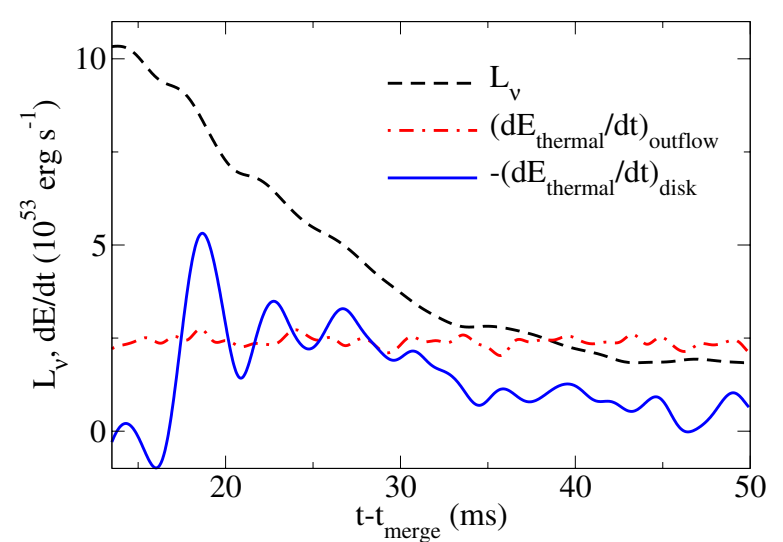

Figure 13. Thermal evolution of the disk during its cooling phase. $L_{v}$ is the neutrino luminosity, $\left(d E_{\text {thermal }} / d t\right)_{\text {outflow }}$ the heat loss due to flows out of the inner and outer boundaries, and $\left(d E_{\text {thermal }} / d t\right)_{\text {disk }}$ the numerical derivative of the total thermal energy in the disk. (The total internal energy is almost exactly $1.15 E_{\text {thermal }}$ throughout the Cowling evolution.) Numerical derivatives and outflow measures are noisy, so the curves have been smoothed in time by convolving with a Gaussian of width $1 \mathrm{~ms}$.

(A color version of this figure is available in the online journal.)

times (as in the figure), the inner disk is nearly in equilibrium, but $Y_{e}>Y_{e}^{\text {eq }}$ in the rest of the disk, so the average $Y_{e}$ decreases slowly. The low-density outer region radiates, and thus responds to emission imbalances, much more slowly. It is still in its initial $Y_{e}$ growth phase. The average energy per neutrino, given by $L_{v_{i}} / R_{v_{i}}$, averaged in time over the disk evolution, is about 12 , 15 , and $19 \mathrm{MeV}$ for $v_{e}, \bar{v}_{e}$, and $v_{x}$ neutrinos respectively; the average neutrino energies are not constant, but decrease at a rate of $1 \mathrm{MeV}$ per $10 \mathrm{~ms}$. When one adds together all four species of $v_{x}$ neutrinos, there are still fewer of them emitted than $v_{e}$ or $\bar{v}_{e}$ neutrinos, but their average energy is sufficiently higher $\left(v_{x}\right.$ are emitted from the hotter regions in the disk interior) that their combined luminosity is slightly larger than $L_{v_{e}}$ and $L_{\bar{v}_{e}}$. The fact that $L_{v_{x}}$ is roughly a quarter of $L_{v_{e}}$ may seem surprising, given that the charged current emissivity in the luminous part of the disk is two orders of magnitude higher than the thermal emissivity. However, charged current processes also dominate the opacity, so that the opacity of the brightest part of the disk is smaller for muon and tau neutrinos $\left(\tau_{\nu_{x}} \lesssim 1\right)$ than for electron (anti)neutrinos $\left(\tau_{v_{e}} \sim 2-5\right)$.

\subsection{Neutrino Cooling Effects}

From Figure 2, we see that the late-time neutrino luminosity is around $2 \times 10^{53} \mathrm{erg} \mathrm{s}^{-1}$ and continuing to drop. During the early disk phase, $\dot{M} \approx 1 M_{\odot} \mathrm{s}^{-1}$, so the accretion efficiency is $\eta=L_{v} / \dot{M} c^{2} \approx 0.1$. The disk remains very nondegenerate throughout the simulation, with the thermal component of the internal energy remaining nearly a constant $85 \%$ of the total internal energy throughout. The actual values of the thermal and internal energies decrease at a rate only about half $L_{v}$ (see Figure 13). The rate of loss of thermal energy due to flow out of the grid, comprised at early times primarily of accretion into the black hole and at late times primarily of disk outflow, is also comparable to $L_{v}$. Thus, these energy sinks are being countered by heat sources sufficient to slow the cooling rate by about a factor of three. Two physical sources of heating in parts of the disk are adiabatic compression (primarily the observed flattening of the disk toward the equator as it cools) and shock heating, the latter presumably occurring in the nonlinearly perturbed regions of the disk. Adiabatic flattening alone is insufficient, since we observe that the rate of total entropy decrease is also much lower than the radiative entropy loss rate. Shock dissipation is the more plausible heat source, since losses in spiral mode energy are of the needed magnitude. A third physical energy source, recombination of nuclei, does not occur during the phase of evolution simulated; the composition of the disk remains $>99 \%$ free nucleons. A nonphysical source of heating would be numerical viscosity. This can only be distinguished from true shock heating by comparing heating rates at different resolutions. We find that L2 and L3 have very similar thermal histories, while L1 cools $30 \%$ more slowly, largely due to a difference in the outflows, although effects of stronger numerical energy dissipation might also contribute. The average specific entropy of the disk provides a clearer sense of whether the remaining matter is heating or cooling. Its evolution is included in Figure 3. We see that, after the initial strong shock heating at disk formation, the entropy begins a slow decrease. The fact that different resolutions show good agreement on the entropy evolution reassures us that numerical dissipation is not dominating the thermal evolution of the disk.

\section{COMPARISON WITH EVOLUTION WITHOUT NEUTRINO COOLING}

At least for the very massive, luminous post-merger system studied here, neutrino cooling and composition changes have a strong effect on the disk structure, even in the early tens of milliseconds. We estimate these effects by comparing simulations carried out with (L1) and without (L1nov) the neutrino leakage source terms added to the fluid equations.

The disk evolved without neutrino cooling develops a significantly higher, and continually growing, entropy. This leads to a larger, more diffuse accretion disk; the maximum density is a factor of 10 lower than for the neutrino-cooled disk, and the density profile lacks the sharp peak in the inner region seen in Figure 8 . The larger extent of the non-cooled disk causes more matter to escape through the outer boundary. During the first $10 \mathrm{~ms}$ after merger, the accretion rate into the black hole for cooled and non-cooled disks is comparable, and so the L1nov disk comes to have a baryonic mass lower by about $40 \%$ than the L1 disk. Again, this is a consequence rather than the cause of the lower density. At later times, the accretion rate of the L1nov disk drops to very low values, but outflow continues.

Because of its lower density, the non-cooled disk actually has a lower average temperature than the cooled disk (4 MeV versus $5 \mathrm{MeV}$ ), despite its higher entropy, during the first $45 \mathrm{~ms}$ after merger. After this, the temperature of the L1 disk drops below that of the L1 nov disk because of neutrino cooling. As expected, neutrino emission strongly affects the evolution of $Y_{e}$. For the L1nov disk, the electron fraction can only change because of the accreted or outflowing matter having $Y_{e}$ that differs from the average, and this turns out to cause only small changes to the average (see Figure 3).

The L1nov disk shows the same persistent perturbations from axisymmetry that are seen in the neutrino-cooled disks. Highenergy, non-equilibrium flow in the inner disk is still seen. This confirms that cooling is not the main driver of perturbations in the cooled disks. We find that the geodesic and equilibrium angular momentum curves show greater deviation for the L1 nov disk - the geodesic curves are essentially the same, but $d L / d r$ is about $25 \%$ shallower for the equilibrium curve in the bulk of the non-cooled disk-indicating that pressure support makes a stronger contribution to the equilibrium of this disk. The entropy 
profiles have the same general shape, and the expected stability properties are similar.

\section{CONCLUSIONS AND FUTURE WORK}

In this paper, we present simulations of a black hole-neutron star binary merger event using a hot nuclear theory-based EOS with neutrino cooling. This allows us to address features of the merger-composition changes, neutrino energy extraction-that are inaccessible to models with any less degree of realism. While important simulations with hot EOS and neutrino leakage have been done in the past, we perform them for the first time in full general relativity. We are thus able to include distinctly relativistic features, such as a high black hole spin.

For this high-spin, moderate-mass system, we find that a significant amount of matter evades prompt capture by the black hole. Roughly $0.08 M_{\odot}$ of low- $Y_{e}$ tidal tail material appears to be dynamically ejected from the system at mildly relativistic speeds. This material will contribute to $r$-process enrichment of the interstellar medium, and may produce quasi-isotropic electromagnetic signals in the form of an optical or infrared kilonova and a radio afterglow, although our simulations do not model such processes. Because the opacity properties of the exotic nuclei formed in the tail are so poorly constrained (although see Kasen et al. 2013 for progress on this problem), quantitative measures of kilonova detectability are subject to large uncertainties. Metzger \& Berger (2012) do, however, define a quantitative figure of merit for the detectability of the radio transient,

$$
\mathrm{FOM}_{\mathrm{rad}}=\left(\frac{E_{\mathrm{ej}}}{10^{50} \mathrm{erg}}\right)\left(\frac{n}{1 \mathrm{~cm}^{-3}}\right)^{7 / 8}\left(\frac{v}{c}\right)^{11 / 4}
$$

where $n$ is the number density of the circumbinary medium. For the ejecta measured in this simulation, and assuming an optimistic circumburst density of $0.1 \mathrm{~cm}^{-3}$ (Berger et al. 2005; Soderberg et al. 2006), we roughly estimate $\mathrm{FOM}_{\mathrm{rad}} \approx 0.15$, which approaches the detection threshold of 0.2 given for the Expanded Very Large Array (EVLA) ${ }^{7}$ at $1 \mathrm{GHz}$, for events within a range of $200 \mathrm{Mpc}$.

Of the matter that remains bound, $0.3 M_{\odot}$ forms a hot, neutrino-optically-thick accretion disk. We find that neutrino cooling has a significant effect on the disk structure; uncooled disks are significantly more extended. Nevertheless, the disk remains hot and thick, with markedly non-Keplerian orbital profiles. The innermost disk configuration appears to be unstable, and order unity deviations from equilibrium and axisymmetry, in the form of millisecond-period spiral features, persist for tens of milliseconds after merger. The neutrino luminosity after merger is initially very high $\left(10^{54} \mathrm{erg} \mathrm{s}^{-1}\right)$, but disk depletion due to accretion and outflow, and energy depletion due to radiation cause the disk to quickly dim. Its luminosity is able to remain above $2 \times 10^{53} \mathrm{erg} \mathrm{s}^{-1}$ for about $50 \mathrm{~ms}$.

Energy deposition efficiencies of $0.2 \%-0.5 \%$ have been seen in prior studies of $v \bar{v}$ annihilation near similar accretions disks (Ruffert et al. 1997; Birkl et al. 2007; Dessart et al. 2009; Harikae et al. 2010). In this particular optimistic case (optimistic because of the energetic, long-lived disk), the $v \bar{v}$ annihilation mechanism may be able to power a fireball with an energy within the range of observed SGRBs. To examine the $v \bar{v}$ efficiency in this scenario, a quantitative analysis is needed, accounting for the spatial and

\footnotetext{
http://www.aoc.nrao.edu/evla/
}

spectral distribution of neutrinos, and the relativistic effects due to the spinning black hole. This could be done, for example, by a ray-tracing solution of the Boltzmann equation.

Additionally, the drop in luminosity at late times is most likely an artifact of the absence in this simulation of physical processes expected to dominate the long-term evolution of the disk. Most obviously, accretion disks like this one will be subject to the magnetorotational instability (Balbus \& Hawley 1991). The MRI can drive vigorous turbulence which, through viscosity or reconnection at small scales, can reheat the disk. Independent of its possible effects on the thermal power available, magnetic forces could make possible Poynting flux-dominated outflows, tapping either the rotational energy of the disk or the spin energy of the black hole.

Although it is undoubtedly interesting, the system studied here is only one case of BHNS merger, and perhaps not a typical one. It will be important to carry out simulations of more systems, covering a range of neutron star masses, black hole masses, and black hole spins. We believe our code captures enough of the relevant physics to be suitable for such explorations; it should certainly improve upon the polytropic neutron star models we and others have previously used for such surveys. Simulations with this level of realism could also be used to study the effects of the different choices of hot EOS used to simulate a merger. Several hot nuclear EOSs have been made publicly available and are suitable for codes like ours (Lattimer \& Swesty 1991; Shen et al. 1998; Shen et al. 2011; Hempel et al. 2012; Steiner et al. 2012).

We hope to make several improvements to future simulations. Much could be gained for nucleosynthesis estimates by tracking the tidal tail out to farther distances and with higher accuracy. To simultaneously resolve outgoing ejecta and the incipient accretion disk is a challenging task for any grid-based code. The solution will likely involve some combination of increased computational resources and improved modeling techniques, such as adaptive meshing or Lagrangian (smoothed particle hydrodynamics) evolutions of the outflow. We are also working to incorporate missing crucial pieces of physics. Magnetic effects require magnetohydrodynamic simulations, while the effects of neutrino heating will only be accessible to our models when we replace the leakage scheme with some form of genuine radiation transport.

We acknowledge helpful discussions with J. Lattimer, L. Roberts, and S. Teukolsky. This research is supported in part by NASA ATP grant No. NNX11AC37G, by the National Science Foundation under grand Nos. PHY-1068243, PHY-106881, PHY-1151197, and AST-1205732, by the Alfred P. Sloan Foundation, and by the Sherman Fairchild Foundation. The computations were performed at Caltech's Center for Advanced Computing Research on the cluster "Zwicky" funded through NSF grant no. PHY-0960291 and the Sherman Fairchild Foundation. Furthermore, computations were performed on the General Purpose Cluster supercomputer at the SciNet HPC Consortium. SciNet is funded by: the Canada Foundation for Innovation under the auspices of Compute Canada; the Government of Ontario; Ontario Research Fund-Research Excellence; and the University of Toronto (Loken et al. 2010).

\section{REFERENCES}

Arnould, M., Goriely, S., \& Takahashi, K. 2007, PhR, 450, 97

Balbus, S. A., \& Hawley, J. F. 1991, ApJ, 376, 214

Bardeen, J. M., Press, W. H., \& Teukolsky, S. A. 1972, ApJ, 178, 347 
Belczynski, K., Taam, R. E., Rantsiou, E., \& van der Sluys, M. 2008, ApJ, 682,474

Berger, E., Price, P., Cenko, S., et al. 2005, Natur, 438, 988

Birkl, R., Aloy, M. A., Janka, H.-T., \& Müller, E. 2007, A\&A, 463, 51

Burrows, A., Reddy, S., \& Thompson, T. A. 2006, NuPhA, 777, 356

Chawla, S., Anderson, M., Besselman, M., et al. 2010, PhRvL, 105, 111101

Demorest, P., Pennucci, T., Ransom, S., Roberts, M., \& Hessels, J. 2010, Natur, 467,1081

Dessart, L., Ott, C. D., Burrows, A., Rosswog, S., \& Livne, E. 2009, ApJ, 690, 1681

Duez, M. D. 2010, CQGra, 27, 114002

Duez, M. D., Foucart, F., Kidder, L. E., Ott, C. D., \& Teukolsky, S. A. 2010, CQGra, 27, 114106

Duez, M. D., Foucart, F., Kidder, L. E., et al. 2008, PhRvD, 78, 104015

East, W. E., \& Pretorius, F. 2012, ApJL, 760, L4

East, W. E., Pretorius, F., \& Stephens, B. C. 2012, PhRvD, 85, 124009

Etienne, Z. B., Faber, J. A., Liu, Y. T., et al. 2008, PhRvD, 77, 084002

Etienne, Z. B., Liu, Y. T., Shapiro, S. L., \& Baumgarte, T. W. 2009, PhRvD, 79, 044024

Etienne, Z. B., Paschalidis, V., \& Shapiro, S. L. 2012, PhRvD, 86, 084026

Faber, J. 2009, CQGra, 26, 114004

Foucart, F. 2012, PhRvD, 86, 124007

Foucart, F., Deaton, M. B., Duez, M. D., et al. 2013, PhRvD, 87, 084006

Foucart, F., Duez, M. D., Kidder, L. E., \& Teukolsky, S. A. 2011, PhRvD, 83, 024005

Foucart, F., Duez, M. D., Kidder, L. E., et al. 2012, PhRvD, 85, 044015

Foucart, F., Kidder, L. E., Pfeiffer, H. P., \& Teukolsky, S. A. 2008, PhRvD, 77, 124051

Freiburghaus, C., Rosswog, S., \& Thielemann, F.-K. 1999, ApJL, 525, L121

Guillot, S., Servillat, M., Webb, N. A., \& Rutledge, R. E. 2013, ApJ, 772, 7

Gundlach, C., \& Please, C. 2009, PhRvD, 79, 067501

Harikae, S., Kotake, K., Takiwaki, T., \& Sekiguchi, Y.-i. 2010, ApJ, 720,614

Harten, A., Lax, P. D., \& van Leer, B. 1983, SIAMR, 25, 35

Hemberger, D. A., Scheel, M. A., Kidder, L. E., et al. 2013, CQGra, 30, 115001

Hempel, M., Fischer, T., Schaffner-Bielich, J., \& Liebendörfer, M. 2012, ApJ, 748,70

Hotokezaka, K., Kiuchi, K., Kyutoku, K., et al. 2013, PhRvD, 87, 024001

Janka, H.-T., Eberl, T., Ruffert, M., \& Fryer, C. L. 1999, ApJL, 527, L39

Jiang, G.-S., \& Shu, C.-W. 1996, JCoPh, 126, 202

Kasen, D., Badnell, N., \& Barnes, J. 2013, ApJ, 774, 25

Kiuchi, K., Sekiguchi, Y., Kyutoku, K., \& Shibata, M. 2012, CQGra, 29, 124003

Korobkin, O., Rosswog, S., Arcones, A., \& Winteler, C. 2012, MNRAS, 426, 1940

Kyutoku, K., Ioka, K., \& Shibata, M. 2013, PhRvD, 88, 041503

Kyutoku, K., Okawa, H., Shibata, M., \& Taniguchi, K. 2011, PhRvD, 84, 064018

Kyutoku, K., Shibata, M., \& Taniguchi, K. 2010, PhRvD, 82, 044049

Lackey, B. D., Kyutoku, K., Shibata, M., Brady, P. R., \& Friedman, J. L. 2013 , arXiv: 1303.6298

Lattimer, J. M., \& Schramm, D. N. 1974, ApJL, 192, L145

Lattimer, J. M., \& Swesty, F. D. 1991, NuPhA, 535, 331
Lee, W. H., Ramirez-Ruiz, E., \& Page, D. 2005, ApJ, 632, 421

Li, L.-X., \& Paczynski, B. 1998, ApJL, 507, L59

Lindblom, L., Scheel, M. A., Kidder, L. E., Owen, R., \& Rinne, O. 2006, CQGra, 23, S447

Liu, X.-D., Osher, S., \& Chan, T. 1994, JCoPh, 115, 200

Loken, C., Gruner, D., Groer, L., et al. 2010, J. Phys.: Conf. Ser., 256, 012026

Lovelace, G., Duez, M. D., Foucart, F., et al. 2013, CQGra, 30, 135004

Metzger, B. D., \& Berger, E. 2012, ApJ, 746, 48

Nakar, E. 2007, PhR, 442, 166

Nakar, E., \& Piran, T. 2011, Natur, 478, 82

Narayan, R., Paczynski, B., \& Piran, T. 1992, ApJL, 395, L83

Nissanke, S., Kasliwal, M., \& Georgieva, A. 2013, ApJ, 767, 124

O'Connor, E., \& Ott, C. D. 2010, CQGra, 27, 114103

Ott, C., Abdikamalov, E., Moesta, P., et al. 2012, ApJ, 768, 115

Özel, F., Psaltis, D., Narayan, R., \& McClintock, J. E. 2010, ApJ, 725, 1918

Pannarale, F., Tonita, A., \& Rezzolla, L. 2011, ApJ, 727, 95

Papaloizou, J. C. B., \& Pringle, J. E. 1985, MNRAS, 213, 799

Pfeiffer, H. P. 2003, PhD thesis, Cornell Univ.

Pfeiffer, H. P., Brown, D. A., Kidder, L. E., et al. 2007, CQGra, 24, S59

Pfeiffer, H. P., Kidder, L. E., Scheel, M. A., \& Shoemaker, D. 2005, PhRvD, 71, 024020

Pfeiffer, H. P., Kidder, L. E., Scheel, M. A., \& Teukolsky, S. A. 2003, CoPhC, 152,253

Piran, T., Nakar, E., \& Rosswog, S. 2013, MNRAS, 430, 2121

Roberts, L. F., Kasen, D., Lee, W. H., \& Ramirez-Ruiz, E. 2011, ApJL, 736, L21

Rosswog, S. 2013, Phil. Trans. R. Soc. A, 371, 20120272

Rosswog, S., \& Davies, M. B. 2002, MNRAS, 334, 481

Rosswog, S., \& Liebendörfer, M. 2003, MNRAS, 342, 673

Rosswog, S., Speith, R., \& Wynn, G. A. 2004, MNRAS, 351, 1121

Ruffert, M., Janka, H.-T., \& Schaefer, G. 1996, A\&A, 311, 532

Ruffert, M., Janka, H.-T., Takahashi, K., \& Schaefer, G. 1997, A\&A, 319, 122

Safronov, V. S. 1960, AnAp, 23, 979

Scheel, M. A., Pfeiffer, H. P., Lindblom, L., et al. 2006, PhRvD, 74, 104006

Seguin, F. H. 1975, ApJ, 197, 745

Sekiguchi, Y., Kiuchi, K., Kyutoku, K., \& Shibata, M. 2011, PhRvL, 107, 051102

Sekiguchi, Y., \& Shibata, M. 2011, ApJ, 737, 6

Shen, G., Horowitz, C., \& Teige, S. 2011, PhRvC, 83, 035802

Shen, H., Toki, H., Oyamatsu, K., \& Sumiyoshi, K. 1998, NuPhA, 637, 435

Shibata, M., \& Sekiguchi, Y. 2012, PThPh, 127, 535

Shibata, M., \& Taniguchi, K. 2011, LRR, 14, 6

Shibata, M., \& Uryu, K. 2006, PhRvD, 74, 121503

Soderberg, A. M., Berger, E., Kasliwal, M., et al. 2006, ApJ, 650, 261

Steiner, A. W., Hempel, M., \& Fischer, T. 2012, ApJ, 774, 17

Steiner, A. W., Lattimer, J. M., \& Brown, E. F. 2010, ApJ, 722, 33

Stephens, B. C., East, W. E., \& Pretorius, F. 2011, ApJL, 737, L5

Taniguchi, K., Baumgarte, T. W., Faber, J. A., \& Shapiro, S. L. 2005, PhRvD, 72, 044008

Timmes, F. X., \& Arnett, D. 1999, ApJS, 125, 277

Toomre, A. 1964, ApJ, 139, 1217 